\title{
Preliminary Benefits Assessment of Traffic Aware Strategic Aircrew Requests (TASAR)
}

\author{
Jeff Henderson $^{*}$ and Husni Idris ${ }^{\dagger}$ \\ Engility Corporation, Billerica, MA, 01821 \\ David J. Wing ${ }^{\ddagger}$ \\ NASA Langley Research Center, Hampton, VA, 23681
}

\begin{abstract}
While en route, aircrews submit trajectory change requests to air traffic control (ATC) to better meet their objectives including reduced delays, reduced fuel burn, and passenger comfort. Aircrew requests are currently made with limited to no information on surrounding traffic. Consequently, these requests are uninformed about a key ATC objective, ensuring traffic separation, and therefore less likely to be accepted than requests informed by surrounding traffic and that avoids creating conflicts. This paper studies the benefits of providing aircrews with on-board decision support to generate optimized trajectory requests that are probed and cleared of known separation violations prior to issuing the request to ATC. These informed requests are referred to as traffic aware strategic aircrew requests (TASAR) and leverage traffic surveillance information available through Automatic Dependent Surveillance - Broadcast (ADS-B) In capability. Preliminary fast-time simulation results show increased benefits with longer stage lengths since beneficial trajectory changes can be applied over a longer distance. Also, larger benefits were experienced between large hub airports as compared to other airport sizes. On average, an aircraft equipped with TASAR reduced its travel time by about one to four minutes per operation and fuel burn by about 50 to 550 lbs per operation depending on the objective of the aircrew (time, fuel, or weighted combination of time and fuel), class of airspace user, and aircraft type. These preliminary results are based on analysis of approximately one week of traffic in July 2012 and additional analysis is planned on a larger data set to confirm these initial findings.
\end{abstract}

\section{Introduction}

$\mathrm{U}$ ser trajectory change requests by the aircrew to air traffic control (ATC) are an everyday part of airspace operations. The change may be a direct route to a downstream waypoint, altitude change, deviation to avoid weather, early descent, turn on course, or many other request scenarios. User requests are made because the current trajectory is not preferred, due to a change in flight priorities or to a change in the environment (e.g., winds or weather). Aircrews currently make these requests mostly without knowledge of the surrounding traffic, and consequently some requests are denied by ATC because of traffic interference. For instance, the requested change may create a traffic conflict (violation of separation requirements) or be too close to the surrounding traffic for the comfort of the air traffic controller and therefore increase controller workload. There are also many reasons why aircrews are not proactively making requests, including aircrews that are unaware of better options or aircrews that are passive due to prior experience with denied requests. While not the only factor, the lack of traffic information may be a significant impediment to users reliably getting trajectory change requests approved and therefore flying more efficiently.

The advent of airborne surveillance, using Automatic Dependent Surveillance - Broadcast (ADS-B) In, provides airspace users with an emerging opportunity to gain benefits by using this new surveillance information. An aircraft operator that equips their aircraft with ADS-B In gains access to high-quality traffic state data of aircraft broadcasting ADS-B Out within reception range. The ground-based services of Automatic Dependent Surveillance -

\footnotetext{
* Senior Research Engineer, 300 Concord Road, Suite 400, AIAA Member

${ }^{\dagger}$ Principal Research Engineer, 300 Concord Road, Suite 400, AIAA Member

${ }^{\ddagger}$ Principal ATM Research Engineer, NASA LaRC, Mail Stop 152, AIAA Member
} 
Rebroadcast (ADS-R) and Traffic Information System - Broadcast (TIS-B) rebroadcast ADS-B Out traffic information of aircraft equipped with Universal Access Transceiver (UAT) and provide radar traffic information of aircraft equipped with a transponder but not ADS-B Out. Using automated processing of these data with onboard conflict detection algorithms, the aircrew would be able to identify trajectory changes that avoid traffic conflicts and other sector constraints, and they could thereby formulate informed requests with increased likelihood of ATC approval. In addition, an automated monitoring capability could notify the pilot when cost-saving trajectory changes that avoid creating conflicts with surrounding traffic are available and should be requested. These informed pilot requests are referred to as traffic aware strategic aircrew requests (TASAR).

Trajectory optimization to meet time or fuel objectives is the focus of several previous studies. An operational evaluation of the Direct-To tool at Fort Worth en route center found an average savings of 9.4 minutes for aircraft on obsolete weather avoidance routes due to direct-to advisories ${ }^{1}$. The average savings is approximately one minute for flights not diverted to longer routes to avoid convective weather or to mitigate congestion. This operational study reflected that conflicts as well as airspace restrictions are considered by controllers after receiving the direct-to advisory. An earlier fast-time simulation study of the Direct-To tool found an average savings of approximately two to three minutes based on applying the tool to 20 en route centers in the contiguous United States ${ }^{2}$. An analysis of wind optimal routing found that filed flight routes take $1.3 \%$ more time on average and require $1.4 \%$ more fuel burned as compared to wind optimal routes ${ }^{3}$. The authors note that these results are generally in agreement with previous studies of wind optimal routes. Special use airspace (SUA) increases flight length, on average, $1.2 \%$ as compared to user preferred more direct routes ${ }^{4}$. However, most of this effect is in the southwest, and in particular, $70 \%$ of increased flight length is attributed to SUAs over Edwards AFB (California), White Sands Missile Range (New Mexico), and Nellis AFB (Nevada). An analysis of flown trajectories found that fuel burned can be reduced by approximately $2.6 \%$ if optimizing both speed and altitude, $1.8 \%$ if optimizing speed only, and $1.2 \%$ if optimizing altitude only as compared to flown trajectories ${ }^{5}$.

The focus of this paper is quantifying the opportunities and benefits of the TASAR concept, which is described in more detail in a companion paper ${ }^{6}$. Benefits were quantified for three types of aircrew requests in this paper: (1) lateral trajectory change after a traffic flow management reroute initiative has ended and aircrews wish to be switched back to more user-preferred routes, (2) lateral strategic trajectory change to avoid convective weather, and (3) a trajectory change (lateral, altitude, or combination lateral and altitude) to switch to a more wind-optimal route. These types of requests were analyzed using a fast-time simulation platform that models the aircrew behavior when generating and sending requests to the controller, as well as the controller evaluation of whether to accept or reject these requests. Operations between origin-destination airport pairs were selected for fast-time simulation on the basis of airport hub size (i.e., large hub, medium hub, small/non-hub) and stage length to represent a range of operations within a fleet. These operations were simulated with and without TASAR equipage to determine user time and fuel benefits as well as potential impacts on conflicts resolved by ATC.

The paper is organized as follows. Section II presents the aircrew request opportunities where TASAR could produce benefits, as well as the potential impacts on the NAS. The quantification method using fast-time simulation of models of aircrew and controller behaviors is presented in Section III. Section IV presents results of the study and Section V includes key conclusions and implications for the concept.

\section{TASAR Opportunities and Benefits}

This section describes the approach for identifying opportunities and benefits for TASAR-equipped aircraft. TASAR-equipped aircraft are those aircraft equipped with TASAR automation that leverages ADS-B In to gain knowledge of surrounding traffic information as well as on-board automation that continuously searches for flight optimization opportunities and determines conflict-free (to a time horizon) trajectory changes that improve aircrew objectives. Opportunities for aircrew requests that are quantified in this paper are first discussed. Then the benefit mechanisms and potential ATC impacts are presented along with metrics to be used to quantify TASAR effects.

\section{A. Identifying TASAR Opportunities}

The focus of this paper is a near-term TASAR deployment. Opportunities for aircrew requests were selected from requests that are relatively common in current operations. One active business jet pilot, one retired commercial pilot, and two retired Oakland Center (ZOA) controllers were consulted to identify operationally acceptable aircrew requests that are commonly used to meet aircrew objectives. One constraint on requests is the practical use of voice communications, which limit route change requests to one or two named waypoints (navigational aids or fixes) before joining the original trajectory downstream, since the voice communication frequency must remain available to ATC for time critical clearances. 
A preliminary quantitative analysis and the previous studies listed in Section I were used to select three types of aircrew requests that are expected to have the highest potential for benefits. This paper focuses on quantifying the benefits of these three types of aircrew requests while recognizing that there are other types of aircrew requests that have opportunities for benefits. Therefore, this analysis represents only part of the expected full benefit of TASAR. The benefits of the following three types of aircrew requests were quantified in this paper:

1) An aircraft is part of a reroute initiative to avoid convective weather or mitigate congestion. Aircraft in these initiatives are sometimes not shifted back to user-preferred routes after the initiative has ended. The aircrew requests a lateral trajectory change direct to a downstream waypoint or changing one or two named waypoints along the trajectory before reconnecting to the route upstream of the arrival fix. Flight operational control, such as the airline operations center, may inform the aircrew that the initiative has ended, or the aircrew may have access to electronic data sources through airborne internet services indicating that the initiative has ended. Alternatively, the aircrew may become aware of granted requests by other aircrews over the voice frequency.

2) An aircraft is impacted by convective weather, and there is sufficient lead time to the convective weather to allow a strategic route change rather than a tactical heading change. The aircrew requests a lateral trajectory change consisting of changing one or two named waypoints along the trajectory before reconnecting to the route upstream of the arrival fix.

3) The aircrew requests a trajectory change (lateral, altitude, or combination lateral and altitude) to switch to a more wind-optimal trajectory. This request for a more wind-optimal trajectory is intended to occur when the aircraft is not impacted by a reroute initiative or convective weather.

Days that contain reroute initiatives, severe convective weather, or are clear of reroute initiatives and convective weather were selected for the simulation described in Section IV. However, since the reroute initiative or convective weather may not have impacted all aircraft on that day, the following logic is used to classify flights into one of the three request types. If an aircraft is part of a reroute initiative that began before the aircraft departed, and the reroute initiative is cancelled or ended before the aircraft reached the arrival fix, then the aircraft is classified as aircrew request type (1) above (even if convective weather is present, since there may be overlap between the three request types). The data source for reroute initiatives is the National Traffic Management Log (NTML), available on the FAA Command Center website (www.fly.faa.gov). If at least one of the alternative routes of the aircraft is projected to enter convective weather, and the aircraft is not part of a reroute initiative that ends or is cancelled, then the aircraft is classified as request type (2). The data source for convective weather is NEXRAD radar mosaic base reflectivity (www.ncdc.noaa.gov). Certain conditions allow aircraft to request a higher altitude to fly over convective weather, but this is not included as part of (2) and so convective weather tops data is not considered. All other aircraft are classified as request type (3). However, there is overlap between the aircrew request types since the aircrew seeks a wind-optimal solution in all cases, but aircrew request type (3) does not have a reroute initiative or severe convective weather impacting the aircraft.

The TASAR onboard decision support automation operates in both an automated mode and a pilot-initiated mode. While in the automated mode, the TASAR automation searches for opportunities and will notify the aircrew if a beneficial trajectory change is identified. In the pilot-initiated mode, the pilot may enter a trajectory change request which is probed for conflicts and potential resolutions identified. No attempt is made to attribute benefits to the automated mode or to the pilot-initiated mode for the three types of aircrew requests listed above.

\section{B. TASAR Benefit Mechanisms}

A benefit mechanism is a causal linkage that converts a function into a benefit by applying the function to mitigate an inefficiency. A more in-depth discussion of benefit mechanisms can be found in a previous paper ${ }^{7}$ Probing desired trajectory changes for possible separation violations with nearby traffic is one function for the benefit mechanism shown in Fig. 1. This function is expected to mitigate the issue that aircrew requests are not always conflict free and are therefore sometimes denied. The other function shown in Fig. 1 is the capability for TASAR-equipped aircraft to generate user-preferred trajectories, which mitigates the issue that the aircraft may not be following their preferred trajectory due to a previous inefficient trajectory assignment, a change in flight priorities, or a change in the environment (e.g., winds or weather). The mechanisms shown between the second and third vertical dashed lines are enabled by these two functions and result in the four benefits shown between the second and third vertical lines in Fig. 1. The four benefits shown are: (1) aircrew is better able to meet their objectives, (2) improved NAS performance, (3) reduced nuisance requests, and (4) reduced conflicts. In order to quantify these benefits, four metrics are shown in boxes with dashed lines in Fig. 1. These metrics are, respective to the four benefits, (1) flight time per aircraft and fuel burned per aircraft for an operator's fleet, (2) NAS-wide effects such as delays, (3) aircrew requests rejected by controller, and (4) conflicts resolved by controller. The results in 
Section IV quantified (1) per-aircraft flight time and fuel burn, (3) aircrew requests rejected, and (4) conflicts relative to a baseline condition without TASAR. NAS-wide metrics (2) are not included in this paper but could be the subject of a future study.

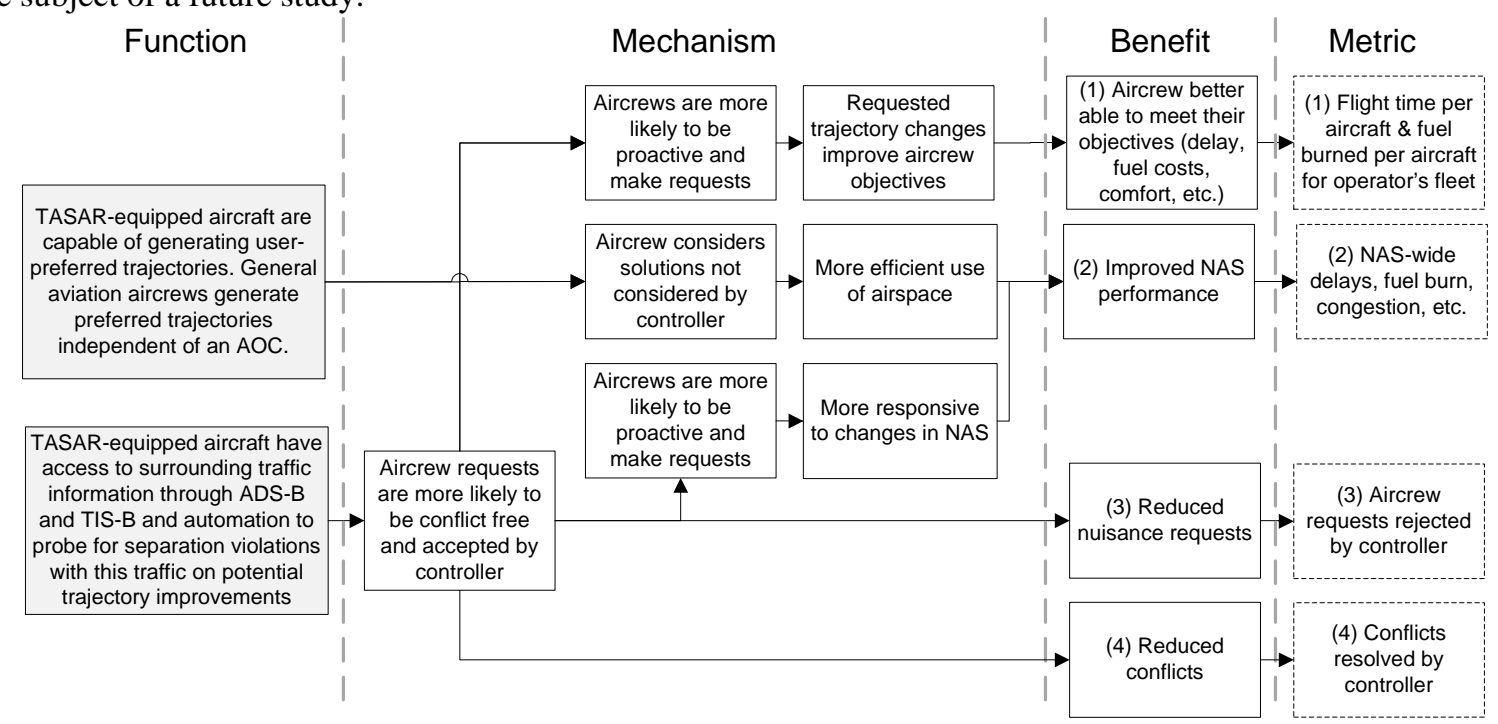

Fig. 1 TASAR benefit mechanisms.

\section{Potential Unintended Impacts of TASAR}

The previous subsection described the potential benefits of TASAR including the potential for reducing NASwide delays, fuel burn, and congestion. However, there is also the potential for TASAR to have unintended impacts. Potential impacts of TASAR could include: (1) the potential for a net increase in conflicts, if the prevention of nearterm conflicts by TASAR results in additional conflicts downstream of the time horizon probed by TASAR and the approving controllers; (2) increased user request denials, if TASAR requests use insufficient margins of separation; (3) increase in airspace complexity, if many deviations from structured routing are requested and approved; and (4) increased controller workload and voice frequency congestion, if TASAR equipage and usage grows too rapidly. The effect on conflicts (1) was analyzed, and results are quantified in Section IV. Analyzing the potential for increased denials (2) would require a human-in-the-loop simulation to address the effect of separation margins on this factor. The effects on airspace complexity (3) and controller workload (4) were not analyzed because this paper focuses on a near-term TASAR deployment with a low percentage of aircraft equipped with TASAR. A future study could consider higher levels of TASAR equipage and the potential to increase airspace complexity (3) and controller workload (4) if several approved requests increase demand and potentially overload a sector far downstream of the sector where the request is granted.

\section{Simulation to Quantify Benefits and Impacts}

This section describes the simulation platform, aircrew model, and controller model used to quantify TASAR benefits and estimate potential impacts on conflicts resolved by ATC. Two retired Oakland Center (ZOA) controllers were consulted to help define operationally acceptable requests used for the aircrew and controller models. The aircrew models use this information to determine which trajectory changes are projected to be operationally unacceptable and will not be sent to the controller as requests, even if the trajectory change is expected to improve aircrew objectives. The controller models estimate operational acceptability to determine whether to accept or reject aircrew requests.

\section{A. Simulation Platform}

An existing simulation platform ${ }^{8,9}$ that connects to the Future ATM Concept Evaluation Tool (FACET) ${ }^{10}$ through an Application Programming Interface (API) was used to model trajectories and airspace structure such as routes and sectors. In the integrated platform, two instances of FACET were used. One instance of FACET, the simulator FACET, was used to model the current state (simulation clock time) of aircraft trajectories. The other instance of FACET, the predictor FACET, was used to model future states of aircraft trajectories to test TASAR aircrew requests for conflicts with surrounding aircraft, conflicts with airspace hazards, and to calculate the impacts of 
TASAR aircrew trajectory change requests on user time and fuel objectives. Both the simulator and predictor instances of FACET were updated at one minute increments. Figure 2 presents an overview of the nine models in the simulation platform used for this study. The first two of these models that use flight plans and radar track inputs (model 1 in Fig. 2) to synthesize trajectories (model 2 in Fig. 2) are discussed in this subsection. The aircrew models (models 3 to 6 above the dashed horizontal line) and controller model (models 7 to 9 below the dashed horizontal line) are discussed in the next subsections.

Model 1: Traffic Generation. Input files to the simulation platform

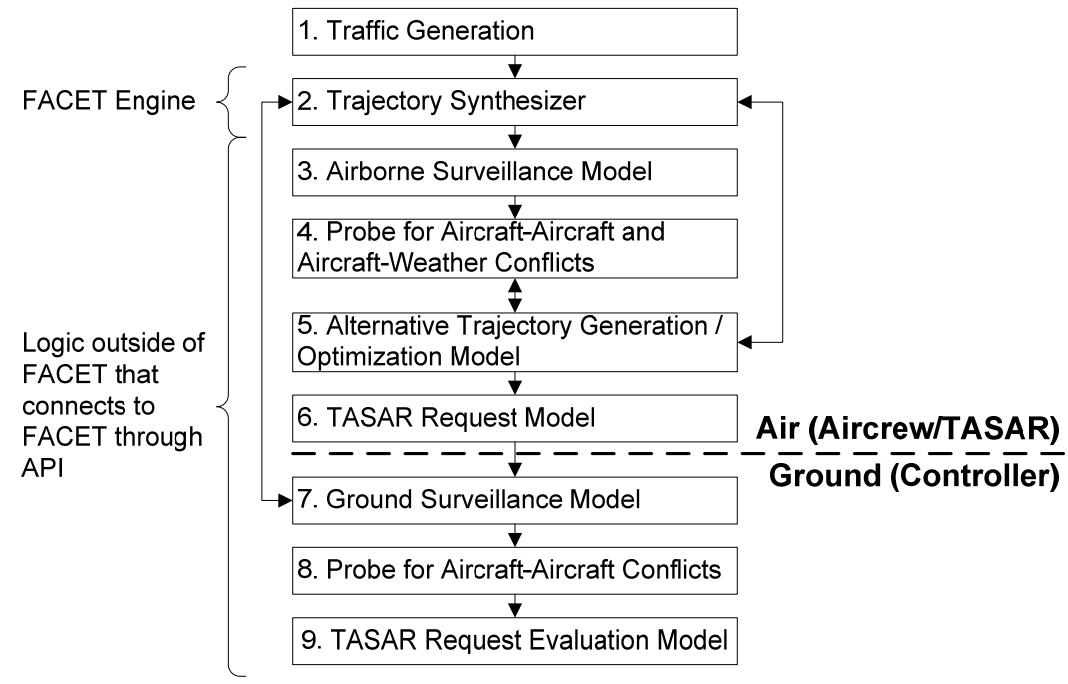

Fig. 2 Models in simulation platform. contain flight plans as well as corresponding historically flown four-dimensional (4D) trajectories. Aircraft were modeled to follow their flown trajectory until an aircrew request is granted. Traffic information was obtained from historical data from the Aircraft Situation Display to Industry (ASDI). Additional input file fields were used to specify assumed TASAR and ADS-B equipage.

Model 2: Trajectory Synthesizer. FACET was configured to predict future aircraft positions differently for historically flown 4D trajectories as compared to alternate trajectories generated by TASAR. Aircraft following their historically flown 4D trajectory did not use aircraft performance or atmospheric models and instead, arrived at the 4D waypoints as specified in the input file. For synthesizing alternate trajectories generated by TASAR, FACET converted the flight plan to a series of latitude and longitude waypoints that were simulated based on aircraft performance models. Wind modeling was based on historical Rapid Update Cycle (RUC) winds data that was read from outside of FACET and was used to update the aircraft groundspeed.

\section{B. Aircrew Model}

Model 3: Airborne Surveillance Model. The airborne surveillance model was used to determine which aircraft that are known to the TASAR automation based on the ADS-B equipage (1090ES, UAT, or none) of the ownship (i.e., the aircraft equipped with TASAR) and the surrounding aircraft. If both the ownship and surrounding traffic aircraft are on the same ADS-B frequency (1090ES or UAT), then a simplified model of $60 \mathrm{nmi}$ omni-directional range was used. Aircraft closer than $60 \mathrm{nmi}$ in any direction was known to TASAR automation through ADS-B In. If the surrounding traffic is equipped with a transponder but not ADS-B Out, then the TASAR automation received information through TIS-B about aircraft within fifteen nmi laterally and 3,000 ft vertically of the ownship if the ownship is at or below the TIS-B 24,000 ft service ceiling. The simulation platform also had models of ADS-R, which applies when the ownship and surrounding traffic were on different frequencies; however, this condition was not part of the scenarios in Section IV. Aircraft on different ADS-B frequencies are expected to occur below FL 180 since aircraft not equipped with 1090ES are not permitted above FL180 after the 2020 ADS-B mandate. Flight plan information was not available through ADS-B for surrounding traffic, and TASAR automation relied exclusively on state information including current position, heading, rate of altitude change, and groundspeed.

Model 4: Probe for Aircraft-Aircraft and Aircraft-Weather Conflicts. The ownship's cleared trajectory, as well as alternative trajectories that improve the aircrew objectives, were probed to an eight minute horizon to determine if there was a conflict with the surrounding traffic. While the minimum separation requirement is five nmi laterally, a conservative ten nmi lateral and 1,000 ft vertical minimum separation shell was used for TASAR automation conflict probing. The conflict probe was used to determine which alternative routes were projected to be operationally acceptable to the controller in addition to improving the objectives of the aircrew. A conflict along the currently assigned trajectory was not considered a trigger to send an aircrew request since it is the role of the controller and not the aircrew to resolve conflicts. 
Alternative trajectories were also probed for conflicts with airspace hazards including special activity airspace (SAA) and severe convective weather. Airspace hazards, either weather or SAA, were defined as polygons with a floor, ceiling, and schedule for activation and deactivation. Polygons were dynamic in the sense that they are active for a defined period of time and then replaced by other polygons at different locations to mimic the motion of convective weather. If the aircraft was predicted (using the FACET predictor instance) to be inside an airspace hazard polygon, then the TASAR automation was modeled to be aware of the airspace hazard conflict. Airspace hazards are probed to a 30 minutes look-ahead horizon.

Model 5: Alternative Trajectory Generation / Optimization Model. The use of voice for aircrew requests limited the alternative lateral trajectories to changing one or two named waypoints before reconnecting to the original trajectory. Computational issues limited the simulations in Section IV to one named waypoint before reconnecting to the original trajectory at the assigned arrival fix which was not modified during the simulation. A bounding box was created for each origin-destination airport pair analyzed in Section IV, and all named waypoints (navigation aids and fixes) inside the bounding box were used to generate alternative trajectories. The bounding box was based on the geographical extent of the flown trajectories between each origin-destination airport pair, so that waypoints further away from either the origin or destination airport than any waypoint on a historically flown trajectory were not considered. Three alternate altitudes were considered at 2000 feet above, 2000 feet below, and 4000 feet below the assigned altitude. FACET does not model dynamic changes to aircraft weight in the simulation so higher altitudes were not considered. FACET models aircraft ceilings in the aircraft performance models based on a typical aircraft weight. Alternative trajectories consisted of lateral changes only, altitude changes only, and combination altitude and lateral changes. The aircraft in the simulation were modeled to follow their historical 4D trajectories once the aircraft passed the arrival fix.

Figure 3 shows an example of five alternative routes for aircraft from Dallas-Fort Worth (DFW) to New York LaGuardia (LGA). This example is for illustrative purposes only, and the simulation results in Section IV contain

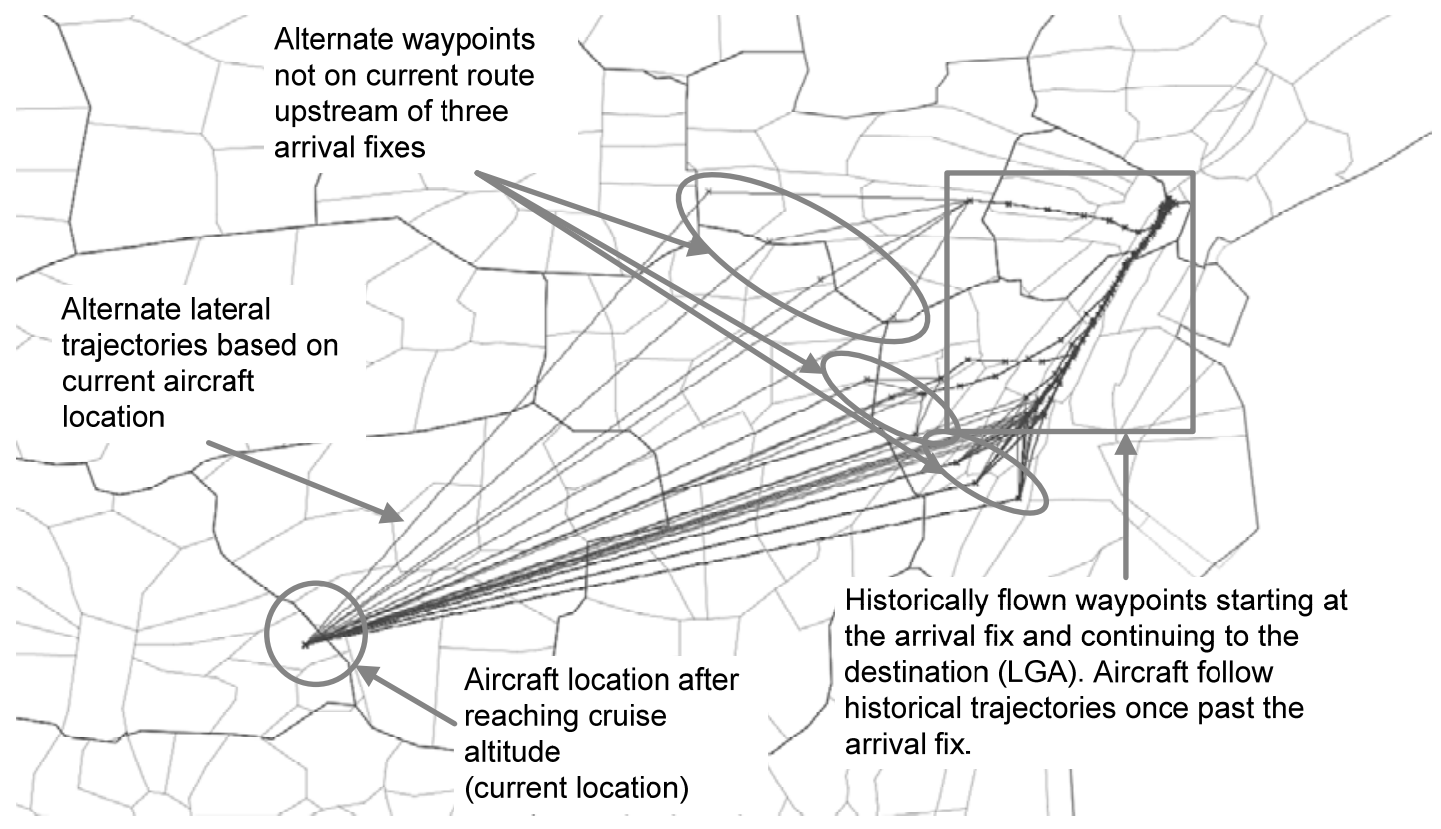

Fig. 3 Example alternative trajectories.

more alternative routes than those shown. The figure shows alternative routes starting from the current aircraft position (circle in lower left) and continuing to either one of four alternative waypoints (circled by ovals) or directly to the arrival fix and then continuing along the flown trajectory to the destination airport.

Model 6: TASAR Request Model. The TASAR request model evaluated potential requests at a specified interval and determined whether or not requests were sent to the controller. The results in Section IV are based on a five minute interval so that requests were generated for all TASAR-equipped aircraft every five minutes and then evaluated by the controller during the same time step. Requests may be evaluated more frequently (e.g., at one minute intervals) by selecting a different value for this parameter. However, there is a tradeoff between computational time and the frequency that requests are generated. 
The decision to make the request was based on aircrew objective improvement and on an estimate of operational acceptability to the controller. If a potential aircrew request does not improve the aircrew objectives, time and fuel in this study, then a request was not sent to the controller. The TASAR logic in the simulation implements filters to prevent the aircrew making requests that would be considered unacceptable to the controller. Requests were not made if any of the following conditions are true:

- Aircraft-aircraft conflict was predicted according to the parameters in model 4.

- Aircraft-airspace hazard conflict was predicted according to the parameters in model 4.

- Aircraft had already made a request to current sector controller. ZOA controllers indicated that multiple requests in a sector is unreasonable and that the aircrew should wait until the next sector to make another request if the initial request is denied.

- Request had no impact on the current sector. Controllers are concerned with their sector and will generally deny requests that do not initiate a trajectory change within the current sector and requires coordination with a downstream sector controller. However, all of the alternative trajectories generated by model 6 involved an immediate trajectory change, so this condition was not enacted during the simulation experiments in Section IV.

- Aircraft was estimated to be in handoff status. ZOA controllers indicated that handoff status is initiated with the downstream controller once the aircraft is within approximately $20 \mathrm{nmi}$ of the sector boundary. Any request received while the aircraft is in handoff status is likely to be met with the response to make the request to the next sector controller.

- Aircraft was on initial climb from origin airport and had not yet reached cruising altitude. Controllers are concerned about potential interference of the departure stream with the arrival stream, so requests are generally denied until the aircraft reaches cruising altitude. However, once at cruising altitude, there is a high opportunity for requests to be granted since this area can become congested and controllers regularly give direct clearances to clear the traffic. This restricting of requests during initial climb generally applies to large hub airports, but the condition was applied in the simulation to all airports to be conservative and for simplicity.

- Aircraft had passed arrival fix that is within $200 \mathrm{nmi}$ of a large hub destination airport. Controllers indicated that aircraft must generally be on their assigned arrival route within $200 \mathrm{nmi}$ of a large hub destination airport.

\section{Controller Model}

Model 7: Ground Surveillance Model. The controller had more information about the surrounding traffic than the TASAR-equipped aircraft including: (1) the flight plans for all aircraft, (2) the ADS-B-equipped aircraft beyond the sixty nmi assumed ADS-B range, and (3) the aircraft not equipped with ADS-B beyond the fifteen nmi assumed TIS-B range.

Model 8: Probe for Aircraft-Aircraft Conflicts. The controller model probed for conflicts based on the same eight minute look-ahead time and ten nmi / 1,000 ft minimum separation shell as the TASAR model 4. However, the controller had more knowledge regarding the surrounding traffic as described in model 7. In this initial study, the controller did not resolve any conflicts. Including controller conflict resolution capabilities is not the primary focus of this analysis and may have interfered with interpretation of time and fuel metrics. The controller model also did not probe for conflicts with convective weather when evaluating aircrew requests.

The baseline conflicts without TASAR requests were estimated since historical trajectories do not contain conflicts that can be directly observed by finding corresponding losses of aircraft separation. Baseline conflicts were estimated similar to conflicts with TASAR requests by projecting the ownship and surrounding traffic trajectories from the current position using the current altitude, current airspeed, and currently assigned route to an eight minute time horizon and determining if there was a conflict that the controller needed to resolve. This estimation disregarded controller actions from the current simulation clock time up to the eight minute look-ahead time horizon in an attempt to exclude historical controller conflict resolution actions. This estimation may have resulted in an underestimation of conflicts for the baseline as compared to conflicts with TASAR since controllers may have modified the historical trajectories based on a longer look-ahead time horizon (e.g., ten minutes) which would not capture all conflicts outside of the eight minute look-ahead time horizon. This longer time horizon that impacts the baseline does not impact trajectories with TASAR requests since the historical controller for the historically flown trajectory would be unaware of the TASAR request made in the fast time simulation. Section IV includes a discussion of the impact of this estimation on the interpretation of conflict results.

Model 9: TASAR Request Evaluation Model. The controller was modeled to reject an aircrew request if any of the following conditions exist: 
- The aircrew request was projected to cause an aircraft-aircraft conflict.

- The aircrew request occurs in a sector that was experiencing traffic exceeding its monitor alert parameter value (i.e., a red sector). This was an attempt to model the phenomenon that, as traffic demand increases in their sector, controllers develop plans to cope with the rising traffic and, unless the request is consistent with the controller plan, the aircrew request is likely to be denied. Under higher traffic levels the aircrew request is less likely to be consistent with the controller plan. This simplified model could be refined to better capture controller behavior under increasing airspace complexity in future studies. However, higher levels of controller workload associated with demand exceeding the monitor alert parameter value when aircrews make requests were relatively infrequent in the scenarios studied in this paper and this lack of a more refined model likely had a negligible impact on the results in Section IV.

- The aircrew request was projected to enter an adjacent red sector. ZOA controllers indicated that controllers are not aware of red sectors elsewhere and will not consider traffic demand in other sectors when evaluating aircrew requests. However, the area manager may instruct the controller not to send traffic through an adjacent sector if the adjacent sector is currently experiencing high traffic. This initial study considered a low level of TASAR equipage, and aircrew requests would be insufficient to overload downstream sectors. However, overloading downstream sectors is a concern under higher levels of TASAR equipage if several aircrews submit requests after, for example, a reroute initiative expires. This factor could be the subject of a future study.

The filters described in TASAR model 6, such as not making multiple requests to the same sector controller, were not applied again in the controller model 9, since these types of requests would not reach the controller in the simulation.

\section{Results}

This section presents the selection of representative airport pairs for network, low cost, regional, and business airspace user operations. Fast-time simulation of flights between these pairs is then used to obtain time, fuel, and other metrics that characterize TASAR requests.

\section{A. Scenarios}

Scenarios were selected for simulation based on classifications of airport hub size and stage length to represent typical operations for different classes of NAS users. Airport hub size was selected as a classification since aircraft operating to and from larger airports are expected to be part of a reroute initiative more frequently and there are more restrictions placed on aircraft as they approach a large hub airport. Stage length was selected as a classification since longer haul flights may be able to apply beneficial trajectory changes over a longer distance, which may result in relatively more benefits per operation than shorter haul flights. The FAA classifications of airports used in this analysis are as follows:

- Large hub - Primary commercial service airport with $1 \%$ or more of total annual passenger boardings per year

- Medium hub - Primary commercial service airport with at least $0.25 \%$ but less than $1 \%$ of total annual passenger boardings per year

- Small hub - Primary commercial service airport with at least $0.05 \%$ but less than $0.25 \%$ of total annual passenger boardings per year

- Non-hub - Primary commercial service and non-primary commercial service airports with at least 2,500 but less than $0.05 \%$ of total annual passenger boardings per year

- General Aviation (GA) - Reliever and general aviation non-primary airports excluding airports with commercial service

The four classes of airspace users listed next (from www.bts.gov) were analyzed to determine the percentage of traffic by type of operation:

- Network carrier - one of the following five carriers: Alaska (ASA), United (UAL), Delta (DAL), US Airways (AWE), and American (AAL)

- Low-cost carrier - one of the following seven carriers: Spirit (NKS), JetBlue (JBU), Allegiant (AAY), Southwest (SWA), Virgin America (VRD), AirTran (TRS), and Frontier (FFT)

- Regional carrier - one of the following six carriers: Horizon (QXE), SkyWest (SKW), American Eagle (EGF), Pinnacle (FLG), ExpressJet (ASQ), and Mesa (ASH)

- Business - NetJets (EJA) operations 
Table 1 Percentage of operations by class of airspace user, airport size, and stage length

\begin{tabular}{|c|c|c|c|c|c|}
\hline $\begin{array}{c}\text { Origin to } \\
\text { Destination }\end{array}$ & $\begin{array}{c}\text { Stage Length } \\
\text { (nmi) }\end{array}$ & Network & Low Cost & Regional & Business \\
\hline \multirow[t]{5}{*}{ Large Hub to Large Hub } & $0+$ & $80.8 \%$ & $50.1 \%$ & $14.6 \%$ & $2.5 \%$ \\
\hline & 0 to 900 & $12.7 \%$ & $10.9 \%$ & $9.3 \%$ & $1.2 \%$ \\
\hline & 900 to 1600 & $24.1 \%$ & $14.1 \%$ & $5.3 \%$ & $0.2 \%$ \\
\hline & 1600 to 2000 & $14.4 \%$ & $6.7 \%$ & $0.0 \%$ & $1.0 \%$ \\
\hline & $2000+$ & $29.7 \%$ & $18.3 \%$ & $0.0 \%$ & $0.0 \%$ \\
\hline \multirow{4}{*}{$\begin{array}{l}\text { Large Hub to Medium } \\
\text { Hub or Medium Hub to } \\
\text { Large Hub }\end{array}$} & $0+$ & $16.3 \%$ & $33.7 \%$ & $31.2 \%$ & $2.5 \%$ \\
\hline & 0 to 700 & $3.2 \%$ & $8.3 \%$ & $14.3 \%$ & $2.3 \%$ \\
\hline & 700 to 1200 & $4.0 \%$ & $11.4 \%$ & $14.8 \%$ & $0.1 \%$ \\
\hline & $1200+$ & $9.1 \%$ & $13.9 \%$ & $2.0 \%$ & $0.1 \%$ \\
\hline \multirow{4}{*}{$\begin{array}{l}\text { Large Hub to Small/Non- } \\
\text { Hub/GA or Small/Non- } \\
\text { Hub/GA to Large Hub }\end{array}$} & $0+$ & $2.8 \%$ & $9.1 \%$ & $50.1 \%$ & $42.8 \%$ \\
\hline & 0 to 300 & $0.4 \%$ & $0.4 \%$ & $8.5 \%$ & $3.8 \%$ \\
\hline & 300 to 600 & $1.2 \%$ & $1.8 \%$ & $24.5 \%$ & $4.1 \%$ \\
\hline & $600+$ & $1.3 \%$ & $6.9 \%$ & $17.1 \%$ & $34.9 \%$ \\
\hline \multirow[t]{3}{*}{ All other operations } & $0+$ & $0.1 \%$ & $7.2 \%$ & $4.1 \%$ & $52.2 \%$ \\
\hline & 0 to 600 & $0.0 \%$ & $4.7 \%$ & $3.4 \%$ & $26.9 \%$ \\
\hline & $600+$ & $0.0 \%$ & $2.5 \%$ & $0.7 \%$ & $25.4 \%$ \\
\hline
\end{tabular}

ASDI flight plan data during the first two weeks of July were analyzed to determine the percentage of operations by different airspace user class in Table 1 . The left two columns in Table 1 are the categories for airport hub size and stage length between the origin airport and destination airport. The right four columns are the classes of airspace users. For example, the first row shows that $80.8 \%, 50.1 \%, 14.6 \%$, and $2.5 \%$ of operations were between large hub airports for network, low cost, regional, and business NAS users respectively. The second row, and all rows with percentages that are not italicized, are percentages based on the specified stage length in the second column.

Table 1 shows that network carriers primarily operated between large hub airports. Low cost carriers had more operations at medium and smaller airports than network carriers. Regional carriers primarily operated between a medium or small airport and a large hub at shorter stage lengths than network or low cost carriers. Business operators had a higher percentage of operations involving non-large hub airports than the other three classes of NAS users.

The airport pairs in Table 2 were selected for analysis since they were representative of the airport hub size and stage length classes in the left two columns of Table 1 . There are other potential factors that may impact opportunities for trajectory improvements, such as congestion around the airport and geographic location in the NAS, but these other factors were not quantified for this initial analysis. The third and fourth columns from the left, labeled airport 1 and airport 2 respectively, are the operations analyzed and includes operations from airport 1 to airport 2 (e.g., ATL to DFW) as well as operations from airport 2 to airport 1 (e.g., DFW to ATL). The second column from the right in Table 2 is the great circle stage length between the airports listed in the third and fourth columns from the left. The rightmost column lists typical aircraft types that operated between the airports in order from most common (top) to less common (bottom). For example, the most common aircraft that operated between DFW and LGA is a Boeing 737.

The following days were analyzed and the results quantified in the next subsections: July $11^{\text {th }} 2012$ (convective weather in the southeast for part of the day), July $12^{\text {th }}$ to $17^{\text {th }}$, 2012(convective weather during parts of most days), July $18^{\text {th }}, 2012$ (convective weather on east coast), July $19^{\text {th }}$, 2012 (convective weather on east coast), and July $20^{\text {th }}$, 2012 (convective weather on east coast and Midwest). Only the ORD-PWM, TEB-CMH, and HPN-SNA airport pairs were analyzed for the July $12^{\text {th }}$ to $17^{\text {th }}$, 2012 date range in order to obtain a larger sample size for these airport pairs.

\section{B. Time and Fuel Metrics}

The time and fuel metrics presented in this subsection were based on $100 \%$ ADS-B out equipage. The effect of ADS-B equipage levels was analyzed in subsection E. Three aircrew objectives are considered in this analysis: (1) minimize time, (2) minimize fuel, and (3) weighted combination of minimizing time (50\%) and minimizing fuel (50\%). This analysis also attempts to determine whether stage length, airport size, or type of request, as defined in Section II, determines which aircraft would receive the highest benefits from TASAR. 
Table 2 Airport pairs for analysis

\begin{tabular}{|c|c|c|c|c|c|}
\hline $\begin{array}{c}\text { Origin to } \\
\text { Destination }\end{array}$ & $\begin{array}{c}\text { Stage Length } \\
\text { (nmi) }\end{array}$ & Airport 1 & Airport 2 & $\begin{array}{c}\text { Pair Stage } \\
\text { Length (nmi) }\end{array}$ & $\begin{array}{c}\text { Typical Aircraft } \\
\text { Types }\end{array}$ \\
\hline \multirow[t]{4}{*}{$\begin{array}{l}\text { Large Hub to Large } \\
\text { Hub }\end{array}$} & 0 to 900 & $\begin{array}{c}\text { Atlanta } \\
\text { Hartsfield- } \\
\text { Jackson (ATL) }\end{array}$ & $\begin{array}{c}\text { Dallas/Fort } \\
\text { Worth (DFW) }\end{array}$ & 635 & $\begin{array}{r}\text { MD-80 } \\
\text { Boeing } 757\end{array}$ \\
\hline & 900 to 1600 & $\begin{array}{c}\text { Dallas/Fort } \\
\text { Worth (DFW) }\end{array}$ & $\begin{array}{l}\text { New York La } \\
\text { Guardia (LGA) }\end{array}$ & 1206 & $\begin{array}{r}\text { Boeing } 737 \\
\text { MD-80 } \\
\end{array}$ \\
\hline & 1600 to 2000 & $\begin{array}{l}\text { Chicago O'Hare } \\
\text { (ORD) }\end{array}$ & $\begin{array}{l}\text { Los Angeles } \\
\text { (LAX) }\end{array}$ & 1515 & $\begin{array}{r}\text { Boeing } 737 \\
\text { Airbus A320 }\end{array}$ \\
\hline & $2000+$ & New York (JFK) & $\begin{array}{l}\text { Los Angeles } \\
\text { (LAX) }\end{array}$ & 2148 & $\begin{array}{r}\text { Airbus A320 } \\
\text { Boeing } 767 \\
\end{array}$ \\
\hline \multirow{3}{*}{$\begin{array}{l}\text { Large Hub to } \\
\text { Medium Hub or } \\
\text { Medium Hub to } \\
\text { Large Hub }\end{array}$} & 0 to 700 & $\begin{array}{c}\text { Chicago O'Hare } \\
\text { (ORD) }\end{array}$ & $\begin{array}{c}\text { St. Louis } \\
\text { Lambert (STL) }\end{array}$ & 224 & $\begin{array}{r}\text { Embraer ERJ-145 } \\
\text { Bombardier CRJ } 700\end{array}$ \\
\hline & 700 to 1200 & Denver (DEN) & Portland (PDX) & 861 & $\begin{array}{r}\text { Airbus A320 } \\
\text { Boeing } 737\end{array}$ \\
\hline & $1200+$ & $\begin{array}{c}\text { Chicago O'Hare } \\
\text { (ORD) }\end{array}$ & Portland (PDX) & 1509 & $\begin{array}{r}\text { Boeing } 737 \\
\text { Airbus A320 }\end{array}$ \\
\hline \multirow{3}{*}{$\begin{array}{l}\text { Large Hub to } \\
\text { Small/Non-Hub/GA } \\
\text { or Small/Non- } \\
\text { Hub/GA to Large } \\
\text { Hub }\end{array}$} & 0 to 300 & $\begin{array}{l}\text { Seattle Tacoma } \\
\text { (SEA) }\end{array}$ & Spokane (GEG) & 194 & DeHavilland Dash 8 \\
\hline & 300 to 600 & $\begin{array}{c}\text { Chicago O'Hare } \\
\text { (ORD) }\end{array}$ & $\begin{array}{l}\text { Rochester } \\
\text { (ROC) }\end{array}$ & 383 & $\begin{array}{l}\text { Embraer ERJ-145 } \\
\text { Embraer ERJ-170 }\end{array}$ \\
\hline & $600+$ & $\begin{array}{c}\text { Chicago O'Hare } \\
\text { (ORD) }\end{array}$ & $\begin{array}{l}\text { Portland Maine } \\
\text { (PWM) }\end{array}$ & 780 & Embraer ERJ-145 \\
\hline \multirow[t]{2}{*}{ All other operations } & 0 to 600 & Teterboro (TEB) & $\begin{array}{l}\text { Port Columbus } \\
\text { Ohio }(\mathrm{CMH})\end{array}$ & 407 & $\begin{array}{r}\text { LearJet } 60 \\
\text { Bombardier CRJ } 200\end{array}$ \\
\hline & $600+$ & $\begin{array}{l}\text { Westchester } \\
\text { County (HPN) }\end{array}$ & $\begin{array}{l}\text { Orange County } \\
\text { (SNA) }\end{array}$ & 2132 & Cessna C750 \\
\hline
\end{tabular}

Figure 4 shows the average time savings per aircraft (y-axis) relative to a baseline without TASAR by stage length (x-axis) and airport size for an aircrew minimizing time. Aircraft traveling between large hub airports (shown as a solid black line) saved approximately 1.5 minutes of time at a $600 \mathrm{nmi}$ stage length and these savings increased to approximately eight minutes at $2000+\mathrm{nmi}$ stage lengths. This trend of increasing time savings as stage length increases also held for the other airport sizes, but the slope is not as steep as the solid black line for aircraft traveling between large hub airports. Plots of average time savings when minimizing fuel and weighted combination of time and fuel objectives showed similar trends, as did plots of average fuel savings for the three types of objectives considered in this analysis.

Trajectories on July $11^{\text {th }}, 2012$ between two airport pairs at approximately $1500 \mathrm{nmi}$ stage length highlighted in Fig. 4 were examined to determine the cause of the difference between the time savings between large hubs (ORDLAX) and between a medium and a large hub (PDX-ORD). Figure 5 shows a comparison between the

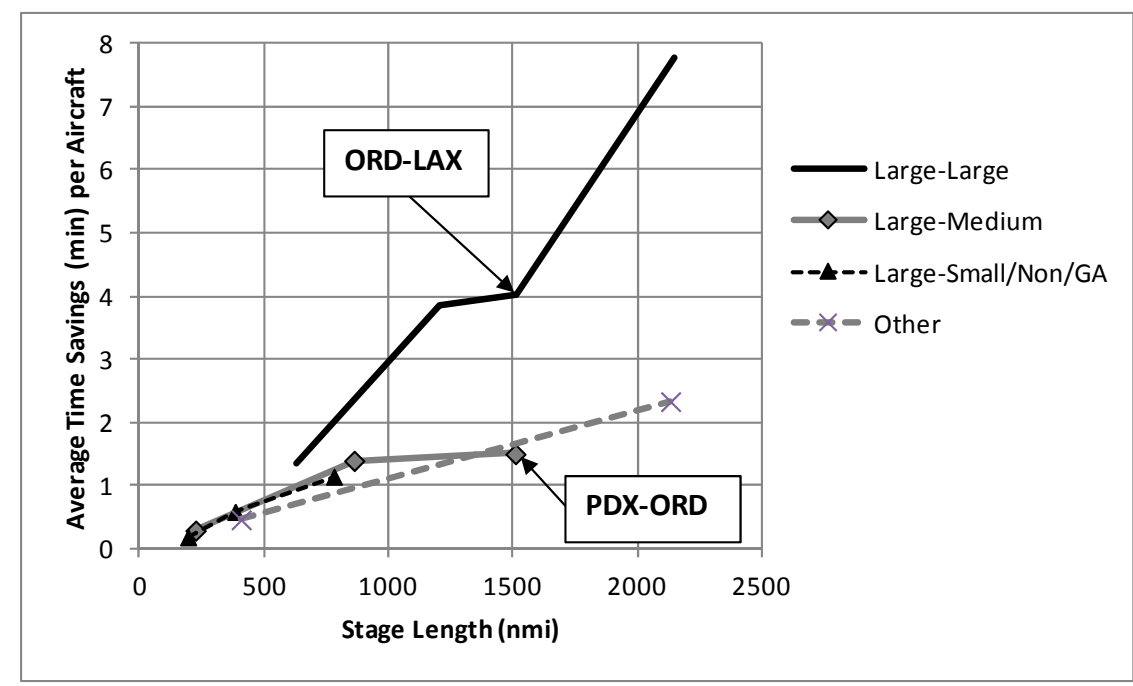

Fig. 4 Average time savings per aircraft by stage length and airport size (Aircrew objective to minimize time and $100 \%$ of traffic equipped with ADS-B Out). 


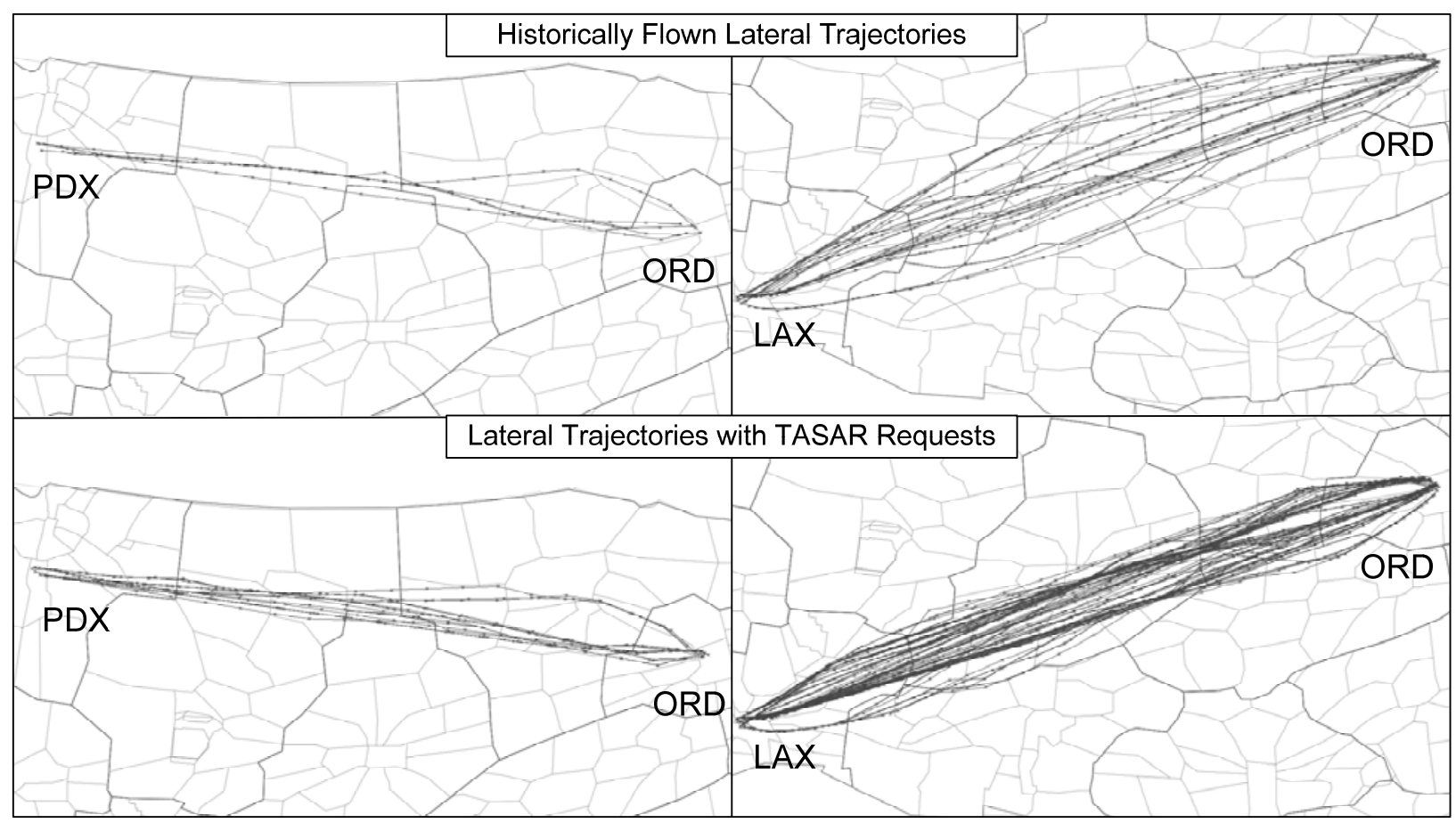

Fig. 5 Comparison of historically flown lateral trajectories and lateral trajectories with TASAR requests for PDX-ORD (left) and LAX-ORD (right). Aircrew objective to minimize time and $100 \%$ of traffic equipped with ADS-B Out.

historically flown lateral trajectories (top) and lateral trajectories with TASAR requests (bottom) for these two aircraft pairs. The spread of the traffic between ORD and LAX was larger than for the traffic between PDX and ORD which allowed for more opportunity for trajectory improvement between large hub airports.

The left plot in Fig. 6 shows the average time savings per aircraft (x-axis) by type of request ( $y$-axis defined in Section II) and was used to help determine what types of requests will result in aircraft experiencing the highest benefit. The solid black bar shows results for a minimize time objective, the light grey bar shows results for a
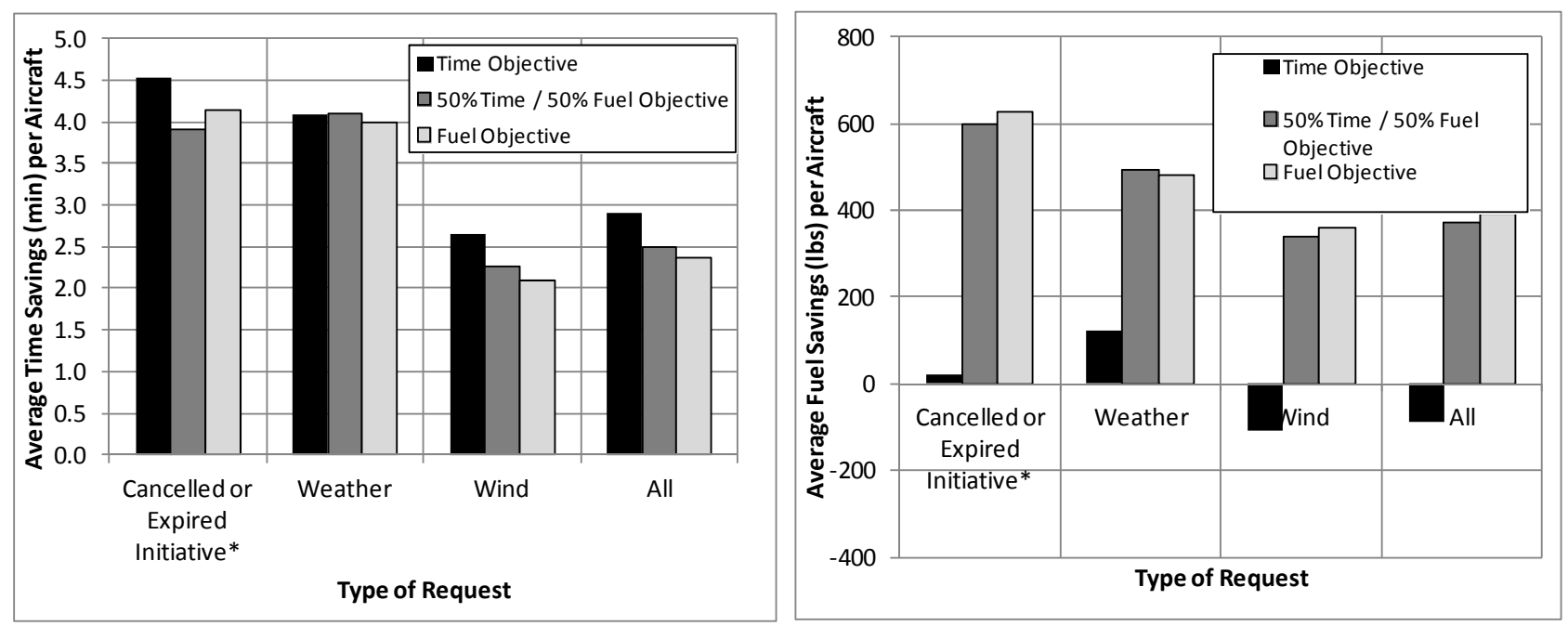

Fig. 6 Average time savings (left plot) and fuel savings (right plot) per aircraft by type of request and aircrew objective (100\% of traffic equipped with ADS-B Out). 
minimize fuel objective, and the middle grey bar represents an intermediate result for a weighted time and fuel objective. For example, selecting a more wind optimal trajectory (third column from the left) resulted in a time savings of approximately $2.7 \mathrm{~min}, 2.2 \mathrm{~min}$, and $2.1 \mathrm{~min}$ for objectives to minimize time, weighted combination of time and fuel, and fuel respectively. Figure 6 shows that there were higher time savings during convective weather conditions as compared to a condition where aircraft are requesting wind-optimal trajectories in the absence of convective weather and reroute initiatives. An insufficient number of flights were involved in a cancelled or expired initiatives to obtain an estimate of time savings for TASAR requests after a reroute initiative is cancelled or expired. A similar plot of average fuel savings per aircraft is shown in the right plot in Fig. 6 with positive values on the y-axis representing fuel savings and negative values representing increased fuel burned. There was increased fuel burned when aircrews request more windoptimal trajectories (third column from the left) to minimize time.

Some aircraft experienced benefits much higher than the average. Figure 8 shows the distribution of time savings for aircraft that traveled between DFW and LGA to meet the objective to minimize time. Each bar represents the count of aircraft (y-axis) with the time savings (x-axis). Even though several aircraft did not benefit (0 time savings), there were aircraft that experienced as much as 13 minutes of time savings. The aircraft with larger improvements had less efficient historically flown

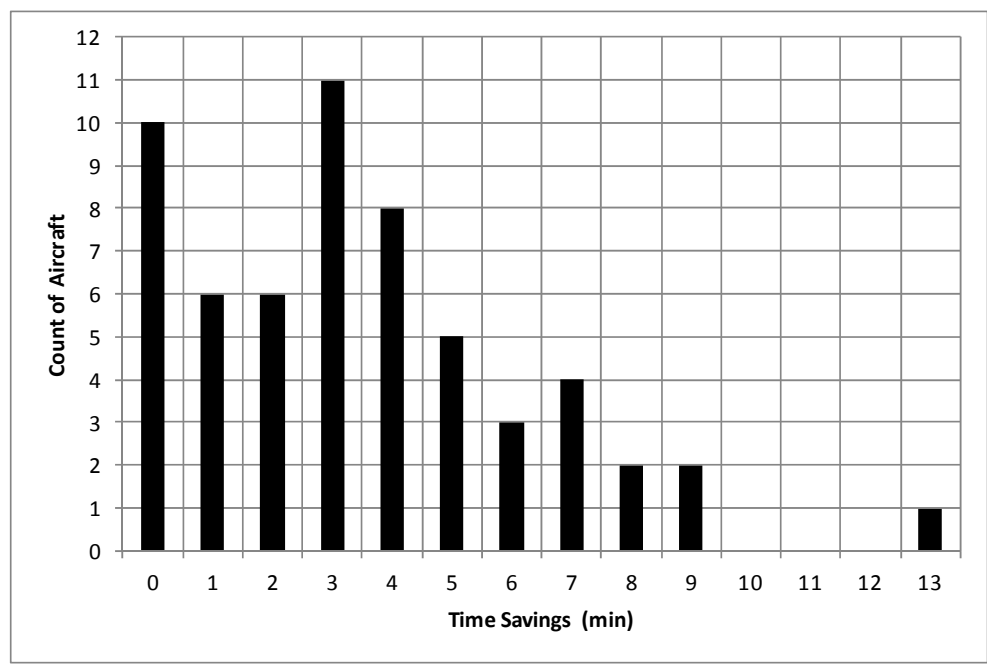

Fig. 8 Distribution of time savings for aircraft traveling from DFW to LGA and from LGA to DFW (minimize time objective and $100 \%$ of traffic equipped with ADS-B Out).

trajectories as compared to aircraft that did not benefit. For example, Fig. 7 shows the historically flown lateral trajectory as well as the trajectory with approved TASAR requests. The example trajectory from LGA to DFW had a 0 min time savings with a small lateral change and no change in altitude as a result of approved TASAR requests. The example trajectory from DFW to LGA had a $13 \mathrm{~min}$ time savings resulting from approved TASAR lateral and altitude trajectory change requests as compared to the

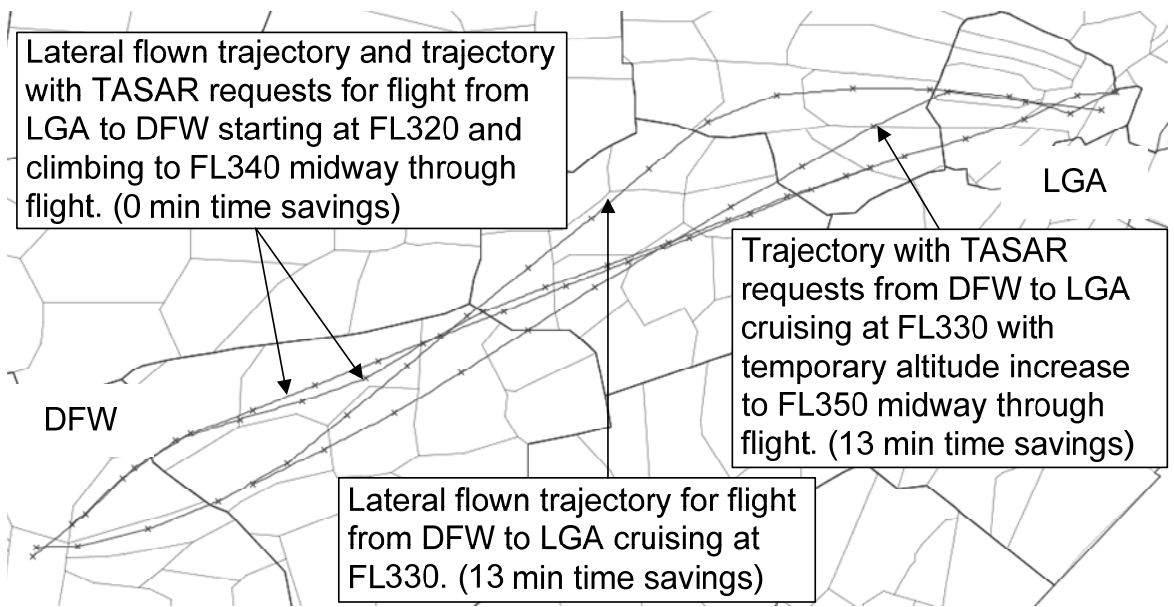

Fig. 7 Example lateral trajectories for an aircraft with 0 min time savings (LGA to DFW) and an aircraft with 13 min time savings (DFW to LGA). historically flown trajectory.

\section{Fleet-Wide per Operation Benefits}

The previous subsection described benefits based on all classes of airspace users and this subsection estimates the benefits by airspace user including network, low cost, regional, and business. Detailed time and fuel savings by aircrew objective, type of request (as defined in Section II), and airport pair are presented in Table 3. The benefits for a specific airspace user could be estimated by finding the airport pairs in Table 3 that most closely matches the 
Table 3 Time and fuel savings by aircrew objective and airport pair. Three types of requests are defined in Section II. TO = time objective, FO = fuel objective, $50 \mathrm{TF}$ = weighted $50 \%$ time $50 \%$ fuel objective

\begin{tabular}{|c|c|c|c|c|c|c|c|c|c|}
\hline \multirow[t]{2}{*}{ Airport 1} & \multirow[t]{2}{*}{ Airport 2} & \multirow{2}{*}{$\begin{array}{l}\text { Type of } \\
\text { Request }\end{array}$} & \multirow[t]{2}{*}{ Flights } & \multicolumn{3}{|c|}{ Time Savings (min) } & \multicolumn{3}{|c|}{ Fuel Savings (lbs) } \\
\hline & & & & TO & FO & 50TF & TO & FO & 50TF \\
\hline \multirow{4}{*}{$\begin{array}{c}\text { Atlanta } \\
\text { Hartsfield- } \\
\text { Jackson (ATL) }\end{array}$} & \multirow{4}{*}{$\begin{array}{c}\text { Dallas/Fort } \\
\text { Worth (DFW) }\end{array}$} & (1) Cx Reroute & 7 & 1.3 & 1.1 & 1.1 & 27 & 212 & 269 \\
\hline & & (2) Weather & 1 & 2.0 & 2.0 & 2.0 & 246 & 246 & 246 \\
\hline & & (3) Wind & 84 & 1.4 & 1.3 & 1.3 & -88 & 328 & 318 \\
\hline & & $(1+2+3)$ All & 92 & 1.4 & 1.3 & 1.3 & -76 & 318 & 313 \\
\hline \multirow{4}{*}{$\begin{array}{c}\text { Dallas/Fort } \\
\text { Worth (DFW) }\end{array}$} & \multirow{4}{*}{$\begin{array}{c}\text { New York La } \\
\text { Guardia } \\
\text { (LGA) }\end{array}$} & (1) Cx Reroute & 25 & 3.5 & 3.4 & 3.3 & -171 & 477 & 467 \\
\hline & & (2) Weather & 6 & 5.7 & 6.0 & 6.2 & 399 & 727 & 749 \\
\hline & & (3) Wind & 28 & 3.8 & 2.8 & 2.5 & -61 & 276 & 253 \\
\hline & & $(1+2+3)$ All & 59 & 3.8 & 3.4 & 3.2 & -61 & 407 & 394 \\
\hline \multirow{4}{*}{$\begin{array}{c}\text { Chicago } \\
\text { O'Hare (ORD) }\end{array}$} & \multirow{4}{*}{$\begin{array}{l}\text { Los Angeles } \\
\text { (LAX) }\end{array}$} & (1) Cx Reroute & 4 & 1.5 & -0.8 & -0.5 & -652 & -53 & -62 \\
\hline & & (2) Weather & 0 & 0.0 & 0.0 & 0.0 & 0 & 0 & 0 \\
\hline & & (3) Wind & 78 & 4.2 & 3.3 & 3.7 & -181 & 565 & 542 \\
\hline & & $(1+2+3)$ All & 82 & 4.0 & 3.1 & 3.5 & -204 & 535 & 513 \\
\hline \multirow{4}{*}{$\begin{array}{l}\text { New York } \\
\text { (JFK) }\end{array}$} & \multirow{4}{*}{$\begin{array}{l}\text { Los Angeles } \\
\text { (LAX) }\end{array}$} & (1) Cx Reroute & 21 & 7.8 & 7.3 & 6.7 & 372 & 1133 & 1050 \\
\hline & & (2) Weather & 1 & 7.0 & 3.0 & 3.0 & -1256 & 229 & 229 \\
\hline & & (3) Wind & 60 & 7.8 & 5.7 & 6.5 & -316 & 1114 & 1119 \\
\hline & & $(1+2+3)$ All & 82 & 7.8 & 6.1 & 6.5 & -151 & 1108 & 1091 \\
\hline \multirow{4}{*}{$\begin{array}{c}\text { Chicago } \\
\text { O'Hare (ORD) }\end{array}$} & \multirow{4}{*}{$\begin{array}{c}\text { St. Louis } \\
\text { Lambert } \\
\text { (STL) }\end{array}$} & (1) Cx Reroute & 0 & 0.0 & 0.0 & 0.0 & 0 & 0 & 0 \\
\hline & & (2) Weather & 0 & 0.0 & 0.0 & 0.0 & 0 & 0 & 0 \\
\hline & & (3) Wind & 38 & 0.3 & 0.2 & 0.2 & 7 & 13 & 13 \\
\hline & & $(1+2+3)$ All & 38 & 0.3 & 0.2 & 0.2 & 7 & 13 & 13 \\
\hline \multirow[t]{4}{*}{ Denver (DEN) } & \multirow{4}{*}{$\begin{array}{l}\text { Portland } \\
\text { (PDX) }\end{array}$} & (1) Cx Reroute & 0 & 0.0 & 0.0 & 0.0 & 0 & 0 & 0 \\
\hline & & (2) Weather & 1 & 1.0 & 3.0 & 3.0 & -78 & 401 & 401 \\
\hline & & (3) Wind & 9 & 1.6 & 1.4 & 2.1 & -536 & 425 & -87 \\
\hline & & $(1+2+3)$ All & 10 & 1.5 & 1.6 & 2.2 & -490 & 422 & -38 \\
\hline \multirow{4}{*}{$\begin{array}{c}\text { Chicago } \\
\text { O'Hare (ORD) }\end{array}$} & & (1) Cx Reroute & 0 & 0.0 & 0.0 & 0.0 & 0 & 0 & 0 \\
\hline & $(\mathrm{PDX})$ & (2) Weather & 0 & 0.0 & 0.0 & 0.0 & 0 & 0 & 0 \\
\hline & & (3) Wind & 33 & 1.4 & 1.2 & 1.2 & -46 & 129 & 119 \\
\hline & & $(1+2+3)$ All & 33 & 1.4 & 1.2 & 1.2 & -46 & 129 & 119 \\
\hline Seattle & Spokane & (1) Cx Reroute & 0 & 0.0 & 0.0 & 0.0 & 0 & 0 & 0 \\
\hline Tacoma (SEA) & (GEG) & (2) Weather & 0 & 0.0 & 0.0 & 0.0 & 0 & 0 & 0 \\
\hline & & (3) Wind & 44 & 0.2 & 0.0 & 0.0 & 2 & 1 & 1 \\
\hline & & $(1+2+3)$ All & 44 & 0.2 & 0.0 & 0.0 & 2 & 1 & 1 \\
\hline Chicago & Rochester & (1) Cx Reroute & 0 & 0.0 & 0.0 & 0.0 & 0 & 0 & 0 \\
\hline O'Hare (ORD) & (ROC) & (2) Weather & 2 & 0.5 & 0.0 & 0.0 & 19 & 21 & 21 \\
\hline & & (3) Wind & 27 & 0.6 & 0.6 & 0.6 & 4 & 34 & 34 \\
\hline & & $(1+2+3)$ All & 29 & 0.6 & 0.5 & 0.6 & 5 & 33 & 33 \\
\hline Chicago & Portland & (1) Cx Reroute & 0 & 0.0 & 0.0 & 0.0 & 0 & 0 & 0 \\
\hline O'Hare (ORD) & Maine & (2) Weather & 0 & 0.0 & 0.0 & 0.0 & 0 & 0 & 0 \\
\hline & (PWM) & (3) Wind & 14 & 1.1 & 0.4 & 0.7 & -42 & 55 & 46 \\
\hline & & $(1+2+3)$ All & 14 & 1.1 & 0.4 & 0.7 & -42 & 55 & 46 \\
\hline Teterboro & Port & (1) Cx Reroute & 2 & 1.0 & 1.0 & 1.0 & 19 & 19 & 19 \\
\hline (TEB) & Columbus & (2) Weather & 0 & 0.0 & 0.0 & 0.0 & 0 & 0 & 0 \\
\hline & Ohio (CMH) & (3) Wind & 22 & 0.4 & 0.6 & 0.6 & 11 & 19 & 19 \\
\hline & & $(1+2+3)$ All & 24 & 0.5 & 0.6 & 0.6 & 12 & 19 & 19 \\
\hline Westchester & Orange & (1) Cx Reroute & 0 & 0.0 & 0.0 & 0.0 & 0 & 0 & 0 \\
\hline County (HPN) & County & (2) Weather & 0 & 0.0 & 0.0 & 0.0 & 0 & 0 & 0 \\
\hline & (SNA) & (3) Wind & 3 & 2.3 & 5.0 & 4.0 & -29 & 105 & 78 \\
\hline & & $(1+2+3)$ All & 3 & 2.3 & 5.0 & 4.0 & -29 & 105 & 78 \\
\hline
\end{tabular}

airport pairs that the operator regularly flies and applying a weighting based on the percentage of operations between the matching airport pair. However, there are many different operators in the NAS that operate between many airport pairs representing a much wider range of variability than could be captured in Table 3. An important consideration is that Table 3 only represents a small subset of traffic days in July 2012 and a much larger sample size would be needed to draw broad generalizations. 
Given these limitations, an initial calculation of the expected per operation benefits of equipping with TASAR is shown in Table 4 based on the class of airspace user percentages in Table 1 and the detailed results in Table 3. For example, Table 1 indicates that $12.7 \%$ of network carrier traffic was between large hubs with stage lengths less than $900 \mathrm{nmi}$. The Table 3 ATL to DFW results are intended to represent traffic between large hubs at stage lengths less than $900 \mathrm{nmi}$. The " $(1+2+3)$ All” row ( $3^{\text {rd }}$ column from left in Table 3$)$ contains all flights for all types of requests and multiplying $12.7 \%$ by 1.4 will provide the ATL to DFW time savings to meet a minimize time objective. The statistics in Table 4 were obtained by continuing this calculation of multiplying the percentages in Table 1 by the corresponding time savings result in Table 3 for each airport pair and then summing all of these results.

However, this method of weighting the results by the percentages in Table 1 may be more appropriate for the time savings metrics rather than for the fuel savings metrics due to differences in aircraft types between the classes of airspace users. The main effect of changing aircraft types is on the fuel burn rate but airspeed is also influenced by aircraft type. For example, the large

Table 4 Time and fuel savings per operation by aircrew objective and class of airspace user. TO = time objective, $\mathrm{FO}=$ fuel objective, $50 \mathrm{TF}$ = weighted $50 \%$ time $50 \%$ fuel objective

\begin{tabular}{|c|r|r|r|r|r|r|}
\hline \multirow{2}{*}{$\begin{array}{c}\text { Class of } \\
\text { Airspace } \\
\text { User }\end{array}$} & \multicolumn{2}{|c|}{ Time Savings (min) } & \multicolumn{3}{|c|}{ Fuel Savings (lbs) } \\
\cline { 2 - 7 } & TO & FO & 50TF & \multicolumn{1}{c|}{ TO } & \multicolumn{1}{c|}{ FO } & 50TF \\
\hline Network & 4.2 & 3.4 & 3.6 & -122 & 575 & 543 \\
\hline Low Cost & 2.9 & 2.5 & 2.6 & -123 & 406 & 344 \\
\hline Regional & 1.0 & 0.8 & 1.0 & -88 & 137 & 66 \\
\hline Business & 1.2 & 1.6 & 1.5 & -22 & 64 & 53 \\
\hline
\end{tabular}

hub to/from medium hub at the 700 to $1200 \mathrm{nmi}$ stage lengths had a significant percentage of regional traffic. However, the Denver (DEN) to/from Portland (PDX) airport pair selected for this airport pair size and stage length mostly consisted of Airbus A320 and Boeing 737 aircraft which are common among network and low cost carriers but not common among regional carriers.

The third column from the left in Table 4 shows that fuel burn increases when aircrews submit requests to minimize time (negative values in Table 4 indicates that time/fuel increases relative to the baseline historically flown trajectory). For this reason, the results based on minimizing fuel or minimizing a weighted $50 \%$ time and $50 \%$ fuel objective may be more appropriate since these two objectives result in both a time savings and a fuel savings. Network carriers saved approximately 3.6 minutes of time per operation and $540 \mathrm{lbs}$ of fuel per operation when minimizing a weighted time and fuel objective. This benefit was higher than the other airspace user classes due to network carriers operating at longer stage lengths between large hub airports than the other airspace user classes. Also, network carriers operated with larger aircraft relative to regional and business airspace users. Other airspace user classes benefited by about one to three minutes per operation.

\section{Conflicts}

There is no expectation that TASAR would increase or decrease conflicts to be resolved by the controller since TASAR only checks for conflicts on potential trajectory changes to an eight minute look-ahead time horizon and TASAR does not probe for conflicts beyond that time horizon. However, there is a concern that TASAR requests will avoid creating conflicts up to the eight minute TASAR conflict probe lookahead time but may inadvertently result in more conflicts for controllers to resolve beyond the eight minute look-ahead time horizon. The baseline conflicts without TASAR requests were estimated similar to conflicts with TASAR requests since historical trajectories do not contain conflicts that can be directly observed by finding corresponding losses of aircraft separation. Average conflicts per aircraft for aircraft equipped with TASAR were lower than the baseline without TASAR for the three types of aircrew objectives shown in Fig. 9 even with the possible underestimation of conflicts for the baseline discussed in Section III. Figure 9 is shown for the traffic between large hub airports

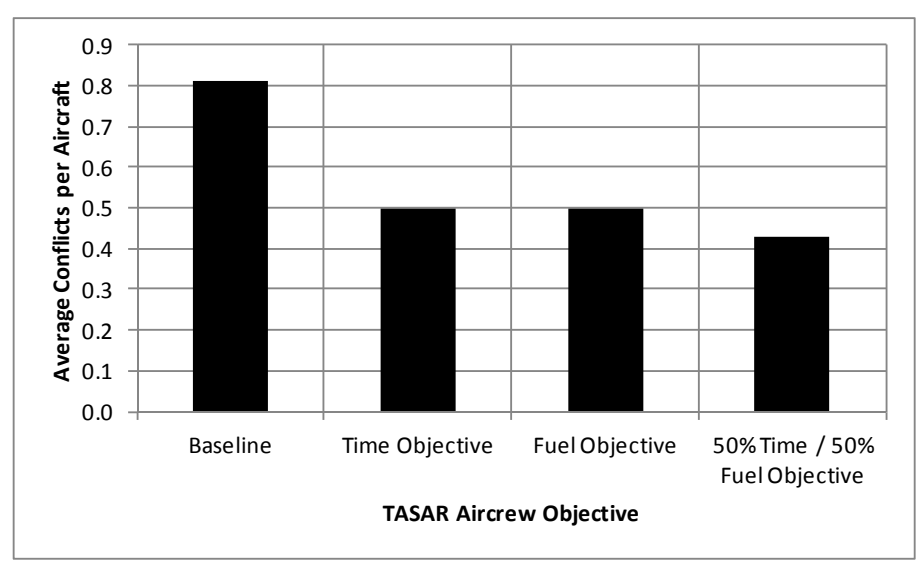

Fig. 9 Average conflicts per aircraft by aircrew objective for aircraft traveling between large hub airports. 
since there are few conflicts between the other airport hub sizes. Also, conflicts during initial climb and conflicts that occur due to merging at the arrival fix are not included in the statistics shown in Fig. 9. The baseline in Fig. 9 shows an average of approximately 0.8 conflicts per aircraft which means that each aircraft analyzed in the baseline without TASAR requests required, on average, 0.8 conflicts to be resolved by the controller. The average conflicts for aircraft equipped with TASAR and the aircrew sending requests were approximately $0.5,0.5$, and 0.4 per aircraft to meet minimum time, fuel, and weighted combination of time and fuel objectives respectively.

The TASAR requests reduced conflicts to be resolved by the controller beyond the eight minute look-ahead time between the large hub airport pairs studied (Fig. 9) by shifting aircraft to altitudes with a lower traffic density. Figure 10 shows the distribution of background traffic at different altitudes (solid black line), the distribution of altitudes for the historically flown trajectories for TASAR equipped aircraft (dashed black line), and the distribution of altitudes for TASAR equipped aircraft sending requests to meet a weighted time and fuel objective (solid grey line with triangle markers). The $\mathrm{x}$-axis is the altitude flight levels and the $y$-axis is the time-weighted percentage of traffic above flight

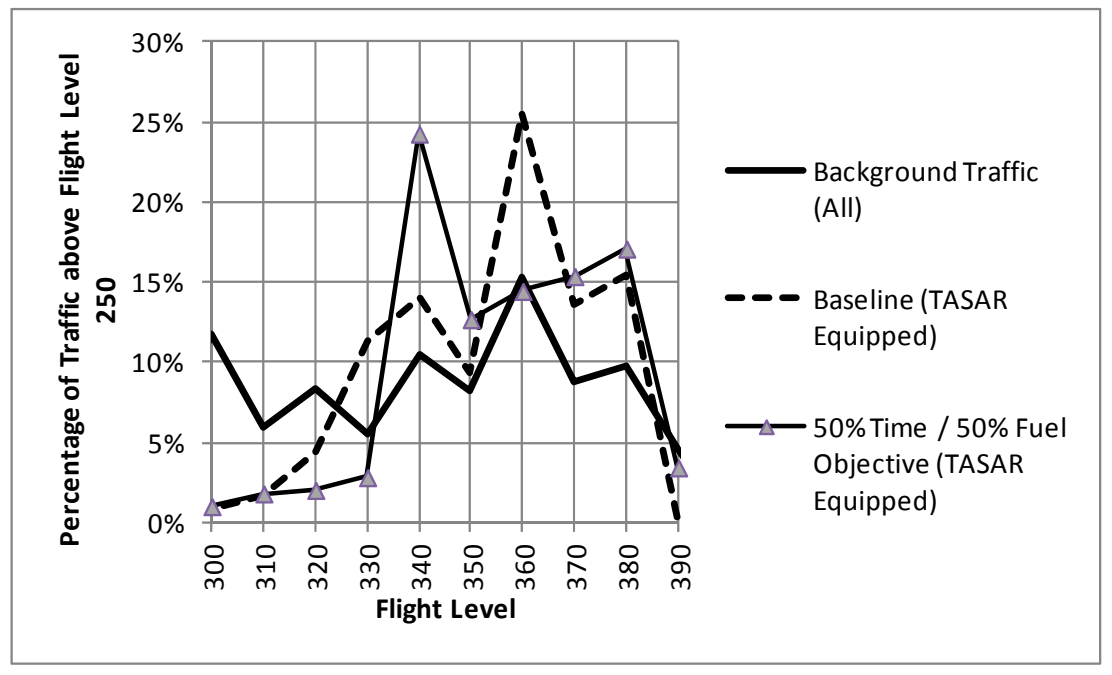

Fig. 10 Distribution of background traffic altitudes above FL 250, historically flown altitude (baseline), and altitude with TASAR requests to meet a weighted time and fuel objective for flights from DFW to LGA. level 250. An example in shifting altitudes can be seen by examining flight levels 360 and 340 that had higher and lower traffic densities, respectively. There was a 33\% drop in background traffic between flight level 360 (15\%) and flight level 340 (10\%) indicating that flight level 340 has a 33\% lower traffic density than flight level 360. The baseline for the aircraft equipped with TASAR indicates that the aircraft spent more time historically at the altitude with higher traffic density (25\% of time at flight level 360) as compared to the altitude with lower traffic density (15\% of time at flight level 340). The TASAR requests changed this trend so that the aircraft spent less time at the flight level 360 higher traffic density altitude (15\%) than at the lower traffic density flight level 340 (25\%).

In general, since the reduction in conflicts was due to aircraft shifting to altitudes with lower traffic densities, the difference between baseline conflicts without TASAR and conflicts for aircraft equipped with TASAR depended on the traffic density at the current altitude and at the requested altitude beyond the TASAR eight minute look-ahead time for conflict probing. If the traffic density at the requested altitude is lower than the traffic density at the current altitude then it is expected that conflicts the controller needs to resolve can be reduced if the request is granted. Alternatively, if the traffic density at the requested altitude is higher than the traffic density at the currently assigned altitude then it is expected that the conflicts the controller needs to resolve will increase if the request is granted.

\section{E. Impact of ADS-B Equipage Level}

One assumption of the TASAR concept is that using traffic information acquired through ADS-B In will increase operational acceptability of aircrew requests to the controller by only issuing aircrew requests that are conflict-free to a time horizon. This subsection presents analysis of the impact of ADS-B Out equipage of surrounding traffic on expected benefits.

Conflicts are one of the reasons that aircrew requests may be rejected and Fig. 12 quantifies the percentage of aircrew rejections due to conflicts as well as other reasons for request rejection by controllers and reasons that requests are not made to the controller due to TASAR estimation of operational unacceptability. The y-axis of Fig. 12 shows the count per aircraft operation of the reasons for rejected requests or requests not made due to TASAR determination of operational unacceptability. Figure 12 is based on $100 \%$ ADS-B Out equipage of traffic for all airport pairs. The left three columns are the conflict rejections per aircraft and the right five columns are the averages per aircraft for conditions where TASAR found a more beneficial trajectory but a request is not sent since 
TASAR determined that request would be operationally unacceptable to the controller. The two columns where conflicts prevent controllers granting aircrew requests, leftmost column and fifth column from the left, total approximately 0.9 per aircraft and are higher combined than any reason other than multiple requests per sector (second from right) at approximately 1.5 indicating that conflicts are a significant factor in determining whether or not aircrew requests will be accepted.

The impact of aircrew requests denied due to conflicts and aircrew requests not sent due to conflicts on time savings is presented in Fig. 11. Figure 11 is for traffic between DFW and LGA only, while Fig. 12 is for all traffic analyzed. The $\mathrm{x}$ axis of Fig. 11 is the percentage of ADS-B Out equipage and the $y$-axes are the average count of unacceptable/ rejected requests (left axis) and the time savings per aircraft (right axis). The dashed grey line is for aircrew requests that are beneficial but are not sent to the controller since TASAR determined that these requests would be operationally unacceptable. This dashed grey line starts at zero at $0 \%$ ADS-B Out equipage and increases to approximately 0.25 per aircraft at $100 \%$ ADS-B Out equipage as the TASAR ownship

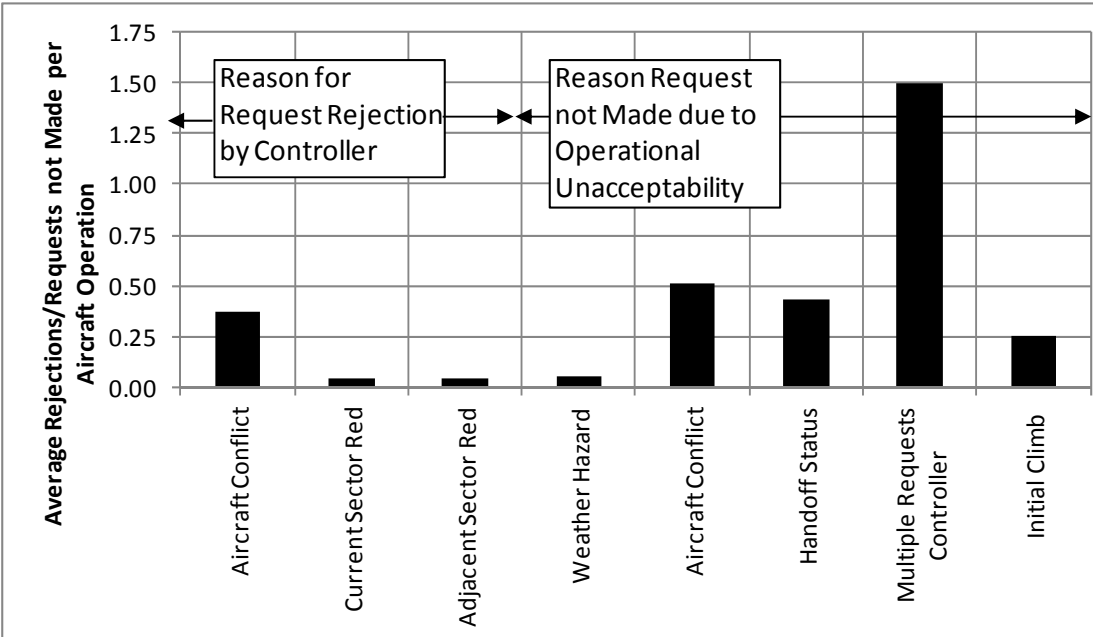

Reason for Request Rejection by Controller or Reason Request not Made due to TASAR Determination of Operational Unacceptability

Fig. 12 Reason for request rejection or request not made due to TASAR determination of operational unacceptability averaged per aircraft operation.

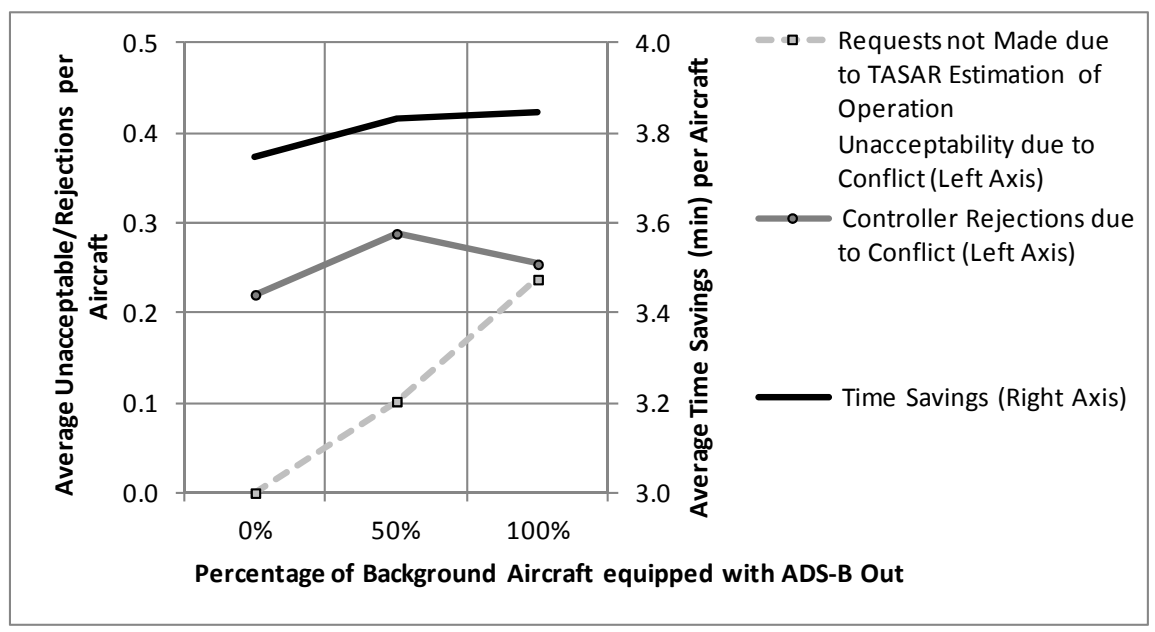

Fig. 11 Impact of Background Traffic ADS-B Equipage on Operational Acceptability, Request Rejections, and Time Savings for Aircrews Minimizing Time (DFW to LGA and LGA to DFW traffic). acquires more surrounding traffic information through ADS-B. However, since TASAR does not have as much information about the aircraft flight plan and future controller intent as does the controller, the TASAR automation did not detect all of the potential conflicts with surrounding traffic as the controller even at $100 \%$ ADS-B Out equipage. This can be seen by examining the solid grey line in Fig. 11 which is the requests rejected by the controller due to conflicts and is approximately horizontal at 0.25 per aircraft and does not equal zero at $100 \%$ ADS-B Out equipage of background traffic. More investigation is needed to determine why the solid grey line does not show a linear trend. However, the surrounding traffic knowledge influences the specific trajectory changes requested in addition to whether or not to make a request which likely has an effect on the controller rejections due to a conflict.

The surrounding traffic equipping with ADS-B Out results in time savings for the TASAR ownship as seen in the solid black line which increases from approximately 3.75 minutes per aircraft at $0 \%$ ADS-B Out equipage to approximately 3.85 minutes per aircraft at 100\% ADS-B Out equipage. A high percentage of ADS-B Out equipage may not be required for TASAR benefits under lower levels of traffic density studied in this paper. Analysis of 
scenarios with higher traffic densities could be used to further investigate the impact of ADS-B Out equipage of background traffic on the expected benefits of TASAR requests.

\section{F. Requests}

The types of requests (lateral, altitude, combined) approved as well as the quantity of aircrew requests are studied in this subsection in order to explain the characteristics of TASAR requests that produced the time and fuel savings described in previous subsections. Figure 13 shows the types of approved requests for each of the three objectives (i.e., time, fuel, and weighted combination). The $\mathrm{x}$-axis is the degree of freedom of the request, and the y-axis is the percentage of requests for that objective. For example, the black column is for the time

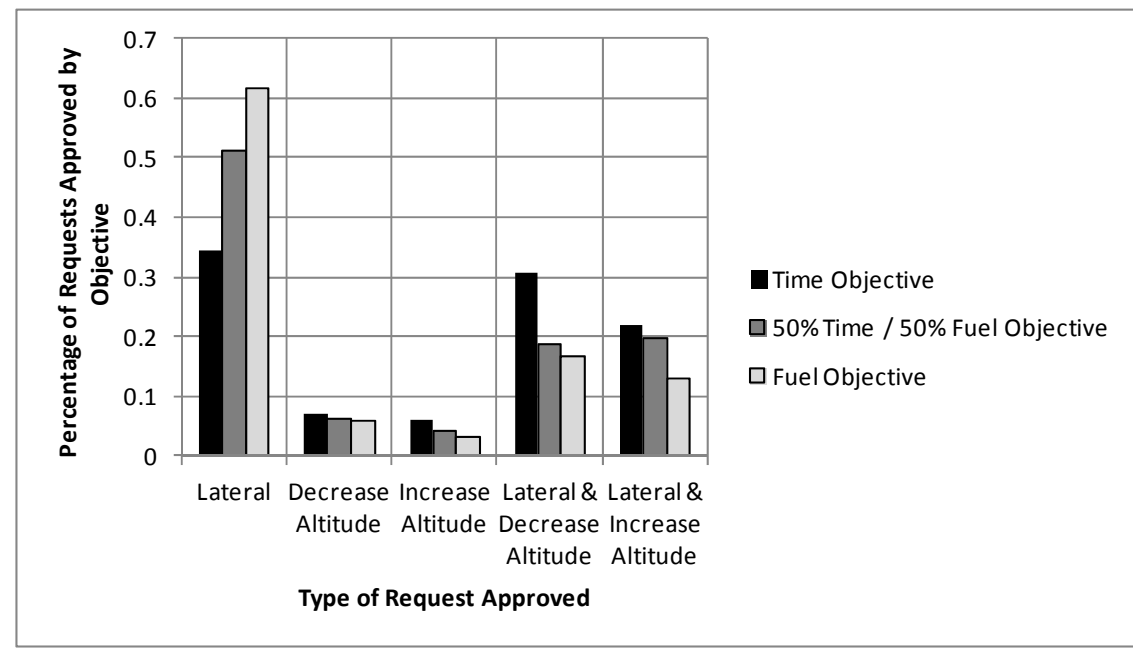

Fig. 13 Percentage of lateral, altitude increase, altitude decrease, and combination requests approved by controller. objective so adding all of the black columns will sum to $100 \%$. Approximately $35 \%$ of aircrew requests to meet the time objective were lateral requests as shown in the left column in Fig. 13. The first lateral request generally resulted in a large lateral shift in the aircraft trajectory while subsequent lateral requests only fine tune the lateral trajectory. So, while a large percentage of approved requests were lateral requests, many of these requests did not have a large impact on the trajectory of the aircraft. A significant portion of requests are a combination lateral and either decrease in altitude ( $30 \%$ of requests to meet a time objective) or increase in altitude (20\% of requests to meet a time objective) indicating that combined lateral and altitude requests were an important source of TASAR benefits. Combined lateral and altitude changes modified the altitude by at least $2,000 \mathrm{ft}$ to have a greater effect than the fine tuning lateral only requests. Aircraft trajectories with TASAR requests generally either (1) decreased after the initial request and do not change cruise altitude to the destination or (2) cycled between decreasing (e.g., change from FL 350 to FL 330) and increasing (e.g., change back from FL 330 to FL 350) altitudes to generate a more wind optimal trajectory.

Figure 14 shows the requests not made due to TASAR determination of operational unacceptability (solid grey line), approved plus rejected requests (solid black line with triangle marker), approved requests only (dashed black line with a rotated square marker), and rejected requests only (dashed grey line with square marker) by stage length. Baseline historical aircrew requests to the controller were not estimated so increased

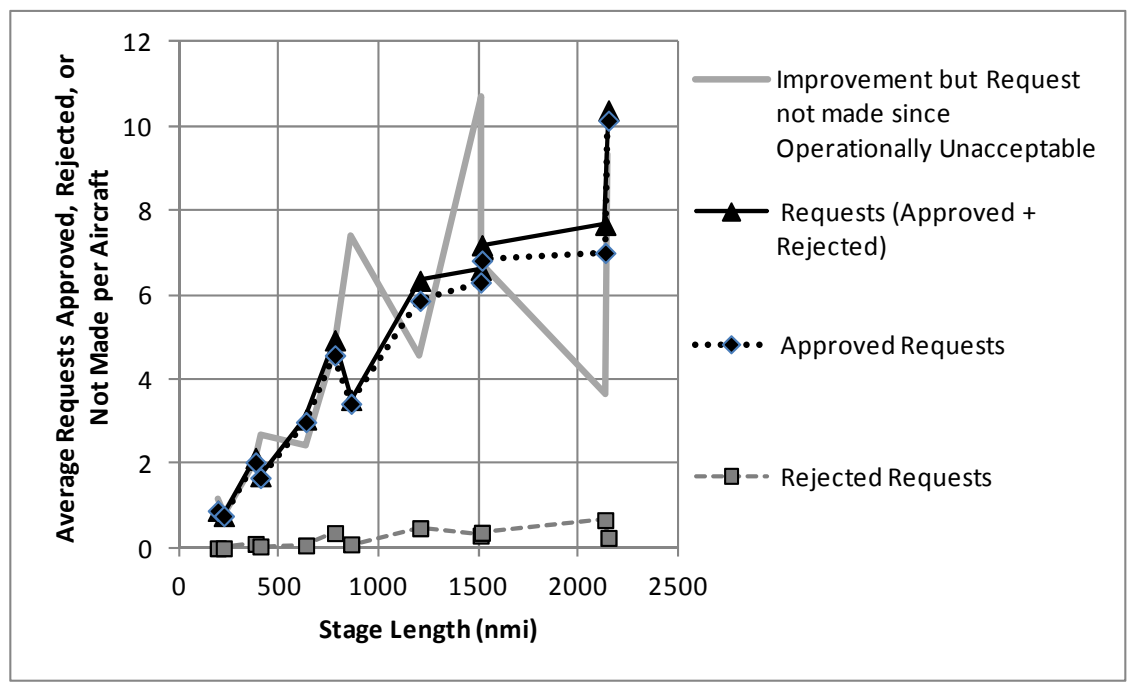

Fig. 14 Requests not made, requests (approved + rejected), approved requests, and rejected requests per aircraft by stage length between airports. 
aircrew requests or decreased aircrew request rejections due to TASAR relative to the baseline were not quantified. However, by examining the figure the following insights were obtained. The figure shows a relatively low percentage of TASAR requests were rejected and, when a beneficial trajectory change was found by TASAR, approximately half of these beneficial requests are not made due to TASAR determination of operational unacceptability and the other half of the beneficial requests are approved. The approved requests increased from approximately 0.5 per operation at a $200 \mathrm{nmi}$ stage length to approximately ten per operation at a $2200 \mathrm{nmi}$ stage length. This relatively large number of lateral requests at $2200 \mathrm{nmi}$ stage length resulted from fine tuning of the lateral trajectory and cycling between increasing and decreasing altitudes en route to generate a more wind-optimal trajectory. Requests could possibly be reduced by refining the TASAR request algorithm in the fast-time simulation platform. It is possible that a more refined algorithm may produce similar benefits with fewer requests.

\section{Conclusions}

This paper quantified the benefits of providing aircrews with on-board decision support to generate optimized trajectory requests that have a high likelihood of ATC approval. These requests are referred to as traffic aware strategic aircrew requests (TASAR) and are probed and cleared of known separation violations prior to issuing the request by using traffic surveillance information available through ADS-B In. Fast-time simulation was used to assess the benefits of three types of aircrew requests: (1) lateral trajectory change after a traffic flow management reroute initiative has ended and aircrews wish to be switched back to more user-preferred routes, (2) lateral strategic trajectory change to avoid convective weather, and (3) a trajectory change (lateral, altitude, or combination lateral and altitude) to switch to a more wind-optimal trajectory. Requests to avoid convective weather (2) produced the highest benefits of the three types of aircrew requests. An insufficient quantity of events where reroute initiatives have ended (1) precluded quantifying the benefits of aircrew requests in this category.

Preliminary results indicated that, in general, benefits increased with longer stage lengths since beneficial trajectory changes can be applied over a longer distance. Also, larger benefits were experienced between large hub airports as compared to other airport sizes. This was largely due to less efficient (from an airspace user point of view) historically flown trajectories between large hub airports and not all flown trajectories operating to or from medium hub or smaller airports have significant room for improvement. On average, aircraft equipped with TASAR, relative to aircraft not equipped with TASAR, saved about one to four minutes of time per operation and about 50 to $550 \mathrm{lbs}$ of fuel per operation depending on the objective of the aircrew (time, fuel, or weighted combination of time and fuel), class of airspace user, and aircraft type. These initial results were based on aircrews requesting lateral only, altitude only, and combination lateral and altitude trajectory changes. The use of combined lateral and altitude trajectory changes provided significant time and fuel benefits since approximately $30 \%$ to $50 \%$ of requests were combination requests depending on the objective of the aircrew.

A key part of the TASAR concept is using traffic information acquired through ADS-B In to improve operational acceptability of aircrew requests to the controller by only requesting trajectory changes that are conflict free to an appropriate time horizon. Fast-time simulation results indicated that conflicts would be the second most common reason why aircrew requests are either not made or rejected by the controller. The most common reason for rejected requests and requests not made was that an aircrew had already made a request to the current sector controller. Initial results indicated that TASAR benefits are immediately achievable under low levels of ADS-B Out equipage. Increased TASAR performance is expected as more aircraft equip with ADS-B Out capability but future work is needed to establish this expected benefit under higher levels of traffic congestion using a more refined TASAR request model that sends fewer requests. Counter to an initial concern regarding a potential net increase in conflicts, TASAR requests were found to reduce conflicts that controllers need to resolve beyond the look-ahead time horizon that TASAR was probing for conflicts. This reduction in conflicts beyond the TASAR conflict probe time horizon was the result of aircrews requesting altitudes with lower traffic densities.

Further analysis is planned on a larger data set to confirm these preliminary time saving, fuel burn savings, and conflict results. Further analysis under higher traffic densities could also help to determine under what conditions TIS-B is sufficient in detecting potential conflicts and to what degree ADS-B Out information received directly from the surrounding traffic is useful in achieving aircrew objectives. Initial results indicate that stage length, airport size, and type of request (e.g., during convective weather) are factors that influence TASAR benefits. Analysis is planned to test the statistical significance of these factors and to establish the relationship between benefits and these factors (e.g., benefits increase linearly with stage length).

\section{Acknowledgments}

This research was funded by NASA under contract NNL12AA06C. 


\section{References}

${ }^{1}$ McNally, D., Engelland, S., Bach, R., Chan, W., Brasil, C., Gong, C., Frey, J., and Vincent, D., "Operational Evaluation of the Direct-To Controller Tool”, $4^{\text {th }}$ USA/Europe ATM R\&D Seminar, Santa Fe, New Mexico, December 3-7, 2001.

${ }^{2}$ Sridhar, B., Chatterji, G.B., and Grabbe, S.R., "Benefits of Direct-To Tool in National Airspace System”, AIAA Guidance, Navigation, and Control Conference and Exhibit, Denver, CO, August 14-17, 2000.

${ }^{3}$ Palopo, K., Windhorst, R., Musaffar, B., and Refai, M., "Economic and Safety Impacts of Flight Routing in the National Airspace System", $7^{\text {th }}$ AIAA Aviation Technology, Integration and Operations Conference (ATIO), Belfast, Northern Ireland, September 18-20, 2007.

${ }^{4}$ Datta, K., and Barrington, C., "Effects of Special Use Airspace on Economic Benefits of Direct Flights", NASA Contractor Report 196704, Contract NAS2-13767, NASA Ames Research Center, Moffett Field, CA, October, 1996.

${ }^{5}$ Lovegren, J.A., and Hansman, R.J., "Estimation of Potential Aircraft Fuel Burn Reduction in Cruise via Speed and Altitude Optimization Strategies”, MIT Report ICAT-2011-03, February, 2011.

${ }^{6}$ Ballin, M.G. and Wing, D.J., "Traffic Aware Strategic Aircrew Requests (TASAR)", AIAA $12^{\text {th }}$ Aircraft Technology, Integration, and Operations Conference (ATIO), Indianapolis, IN, September 2012.

${ }^{7}$ Idris, H., Evans, A., Krozel, J., Doble, N., Eckhause, J., and Hemm, R., "Benefit Mechanisms of Enhanced Collaboration in Tactical Traffic Flow Management”, AIAA Aircraft Technology, Integration, and Operations Conference. Wichita, KA, September 2006.

${ }^{8}$ Henderson, J., Idris, H., Ferguson, S., Kicinger, R., and Krozel, J., "Service Provider Feedback to Support Increased User Traffic Flow Management Responsibility", AIAA $10^{\text {th }}$ Aviation, Technology, Integration, and Operations Conference, Fort Worth, TX, September 13-15, 2010.

${ }^{9}$ Henderson, J., Idris, H., Ferguson, S., Kicinger, R., and Krozel, J., "User and Service Provider Collaboration on Flight Route and Delay under Uncertainty”, AIAA Guidance, Navigation, and Control Conference, Toronto, Canada, August 2010.

${ }^{10}$ Bilimoria, K. D., Sridhar, B., Chatterji, G., Sheth, K. S., and Grabbe, S., "FACET: Future ATM Concepts Evaluation Tool,” Air Traffic Control Quarterly, Vol. 9, No. 1, 2001, pp. 1-20. 